\title{
BOUNDARY VALUE METHODS FOR CAPUTO FRACTIONAL DIFFERENTIAL EQUATIONS*
}

\author{
Yongtao Zhou, Chengjian Zhang ${ }^{1)}$ and Huiru Wang \\ School of Mathematics and Statistics, Huazhong University of Science and Technology, \\ Wuhan 430074, China; \\ Hubei Key Laboratory of Engineering Modeling and Scientific Computing, \\ Huazhong University of Science and Technology, Wuhan 430074, China \\ Email:yongtaozh@126.com, cjzhang@hust.edu.cn, hrwang2013@163.com
}

\begin{abstract}
This paper deals with the numerical computation and analysis for Caputo fractional differential equations (CFDEs). By combining the $p$-order boundary value methods (B$\mathrm{VMs}$ ) and the $m$-th Lagrange interpolation, a type of extended BVMs for the CFDEs with $\gamma$-order $(0<\gamma<1)$ Caputo derivatives are derived. The local stability, unique solvability and convergence of the methods are studied. It is proved under the suitable conditions that the convergence order of the numerical solutions can arrive at $\min \{p, m-\gamma+1\}$. In the end, by performing several numerical examples, the computational efficiency, accuracy and comparability of the methods are further illustrated.
\end{abstract}

Mathematics subject classification: 34A08, 34K28, 65L20.

Key words: Fractional differential equations, Caputo derivatives, Boundary value methods, Local stability, Unique solvability, Convergence.

\section{Introduction}

In this paper, we consider the following initial value problems of CFDEs

$$
y^{\prime}(t)=f\left(t, y(t),{ }_{t_{0}}^{C} D_{t}^{\gamma} y(t)\right), \quad t \in\left[t_{0}, T\right] ; \quad y\left(t_{0}\right)=y_{0},
$$

where $f:\left[t_{0}, T\right] \times \mathbb{R}^{d} \times \mathbb{R}^{d} \rightarrow \mathbb{R}^{d}$ is a given sufficiently smooth function, $y_{0} \in \mathbb{R}^{d}$ is an assigned initial value and ${ }_{t_{0}}^{C} D_{t}^{\gamma} y(t)$ is the $\gamma$-order Caputo derivative of the unknown function $y(t)$ defined by (cf. $[30,32,36])$

$$
{ }_{t_{0}}^{C} D_{t}^{\gamma} y(t)=\frac{1}{\Gamma(1-\gamma)} \int_{t_{0}}^{t} \frac{y^{\prime}(v)}{(t-v)^{\gamma}} d v, \quad 0<\gamma<1 .
$$

The model (1.1) has a wide application in science and technology. For example, in McKee [28] and McKee \& Stokes [29], the diffusion of discrete particles in a turbulent fluid is modeled by the so-called Basset equation:

$$
y^{\prime}(t)=f(t, y(t))+c(t) \int_{t_{0}}^{t} \frac{y^{\prime}(v)}{(t-v)^{\gamma}} d v+g(t), \quad t \in\left[t_{0}, T\right] ; \quad y\left(t_{0}\right)=y_{0},
$$

where $f(t, y(t)), c(t)$ and $g(t)$ are the assigned functions. An extended Basset equation

$$
y^{\prime}(t)=f(t, y(t))+\frac{1}{\Gamma(1-\gamma)} \int_{t_{0}}^{t} \frac{k\left(t, v, y^{\prime}(v)\right)}{(t-v)^{\gamma}} d v, \quad t \in\left[t_{0}, T\right] ; \quad y\left(t_{0}\right)=y_{0},
$$

\footnotetext{
* Received November 12,2018 / Revised version received June 3, 2019 / Accepted July 22, 2019 /

Published online December 6, 2019 /

1) The corresponding author.
} 
can be found in Brunner \& Tang [7] and Hairer \& Maass [14]. Another example is the Babenko's model describing the gas pressure in a fluid (cf. [3]) which is given by

$$
\left\{\begin{array}{l}
\frac{\partial}{\partial t}\left[V_{0} g(t / \theta) P(t, 0) \frac{M}{R T}\right]=\left.F \mathcal{D} \frac{\partial C}{\partial x}\right|_{x=0}, \\
-\left.\sqrt{\mathcal{D}} \frac{\partial C}{\partial x}\right|_{x=0}={ }_{0}^{C} D_{t}^{1 / 2}[C(t, 0)-C(0, x)], \quad t \in[0, \theta] \\
P(t, 0)=\kappa C(t, 0), \quad P(0, x)=\kappa C(0, x), \quad x \in[0, \infty)
\end{array}\right.
$$

where $V_{0}$ is the initial gas volume, $\theta$ is the time of the gas compression to zero volume, $g(t / \theta)$ is the function reflecting the change of gas volume with $g(0)=1$ and $g(1)=0, M, R, \mathcal{D}, F$ denote the gas molar weight, universal gas constant, diffusion coefficient of gas in the fluid and contact surface between the gas and the fluid, respectively, $\kappa$ is the Henry's constant, $C(t, x)$ is the gas concentration and $P(t, x)$ is the unknown gas pressure. The gas temperature $T$ is assumed to be constant. From the problem (1.5), we can obtain the following initial-value problem for determining the dimensionless gas pressure $p(t) \equiv p(t, x)=\frac{P(t, x)}{P(0, x)}$ near the contact surface:

$$
\frac{d}{d t}(g(t) p(t))+\lambda_{0}^{C} D_{t}^{1 / 2}[p(t)-1]=0, \quad t \in[0,1] ; \quad p(0)=1
$$

Let $y(t)=p(t)-1, G(t)=g(t) / g^{\prime}(t)$ and $\hat{G}(t)=\lambda / g^{\prime}(t)$. Then (1.6) can be written as a FDE of the form (1.1):

$$
G(t) y^{\prime}(t)+\hat{G}(t){ }_{0}^{C} D_{t}^{1 / 2} y(t)+y(t)=-1, \quad t \in[0,1] ; \quad y(0)=0 .
$$

Besides the above real models, with the semi-discrete method for the spatial variable $x$, which is also called method of lines, the following fractal mobile/immobile transport models (cf. [26,33]):

$$
\left\{\begin{array}{l}
a_{1} \frac{\partial u(x, t)}{\partial t}+a_{2}{ }_{0}^{C} D_{t}^{\gamma} u(x, t)=a_{3} \frac{\partial^{2} u(x, t)}{\partial x^{2}}+f(x, t), \quad(x, t) \in[a, b] \times\left[t_{0}, T\right], \\
u(x, 0)=\varphi_{0}(x), \quad x \in[a, b], \\
u(a, t)=\phi_{1}(t), \quad u(b, t)=\phi_{2}(t), \quad t \in\left[t_{0}, T\right]
\end{array}\right.
$$

can be transformed into (1.1). A detailed description for this approach refers to Example 6.2. Moreover, some other fractional partial differential equations, such as fractional reactionsubdiffusion equation (cf. [21,24]), fractional cable equation (cf. [25]) and the equations in references $[30,32,36]$, can also be cast into (1.1) by the method of lines.

In contrast to the classical regular Volterra integro-differential equations, the CFDEs have the weakly singular factor $(t-v)^{-\gamma}(0<\gamma<1)$, which leads to the difficulties to obtain the solutions of the equations. Hence, developing various numerical methods for CFDEs becomes an important issue. In [28,29], for Basset equation (1.3), McKee and Stokes proposed the product integration methods based on backward difference interpolation. Subsequently, for the extended Basset equations (1.4), Brunner and Tang [7] constructed the polynomial spline collocation methods and Hairer and Maass [14] presented the fractional linear multistep methods. As to the other related researches for CFDEs, the readers can find them in $[22,26,30,32,36]$ and the references therein. It should be pointed out that, most of the existed numerical methods for CFDEs are presented for the regularity problems (see e.g. [8,22, 27]). However, in general, the solutions of problems (1.1) have the weak singularity at initial point. Hence, it is necessary to consider some computational techniques to treat this issue in order to obtain the expected 
accuracy of the solution. For this, several approaches have been proposed. For example, in $[24,34]$ the authors used the nonuniform grids to keep errors small near the singularity, and in $[9,17]$ the authors employed the correction terms to restore the theoretical accuracy.

In recent years, due to the fact that BVMs and their block schemes have better stability behavior than the usual linear multistep methods, they have been applied widely to solve various initial and boundary value problems (see e.g. $[1,2,4-6,10-12,15,16,18,19,38-44]$ ). These researches devoted mainly to the regular equations excepting those in Aceto, Magherini and Novati $[1,2]$, where the authors extended the generalized Adams methods to solve the following fractional differential equations:

$$
{ }_{t_{0}}^{C} D_{t}^{\gamma} y(t)=f(t, y(t)), \quad t \in\left[t_{0}, T\right], 0<\gamma<1 ; \quad y\left(t_{0}\right)=y_{0} .
$$

It is remarkable that model (1.7) belongs to integral equations because it does not contain any derivative. This shows that model (1.7) is different from CFDEs (1.1). In fact, the research for (1.1) has a greater challenge since it contains both fractional derivative and integer derivative. As we know, up to now, no result has been presented for the BVMs applied to CFDEs (1.1). Hence, in the present paper, we will extend the underlying BVMs to solve (1.1).

The paper is organized as follows. In Section 2, we consider the Lagrange interpolation for $\gamma$-order $(0<\gamma<1)$ Caputo derivatives and investigate its local truncation error. In Section 3, by combining BVMs with the Lagrange interpolation, we derive a class of extended BVMs to solve (1.1). In Section 4, we analyze the local stability and unique solvability of the extended BVMs. In Section 5, under the suitable conditions, we prove that the convergence order of the extended BVMs for (1.1) can arrive at $\min \{p, m-\gamma+1\}$, where $p$ and $m$ are the local order of the extended BVMs and the degree of the Lagrange interpolation, respectively. In Section 6 , some numerical examples are given to illustrate the computational efficiency, accuracy and comparability of the methods.

\section{The Lagrange Interpolation for Caputo Derivatives}

Let $m$ and $N$ be two assigned positive integers, $y(t) \in C^{(m+1)}\left(\left[t_{0}, T\right]\right), t_{n}=t_{0}+n h(n=$ $0,1, \ldots, N)$ and $h=\frac{T-t_{0}}{N}$. Define the following sets:

$$
\begin{aligned}
& \mathcal{A}_{i j}=\{a \mid a \in[0, j], a \neq i, a \in \mathbb{Z}\}, \quad \mathcal{B}_{i j}=\{b \mid b \in[-j, 0], b \neq i-j, b \in \mathbb{Z}\}, \\
& \mathcal{C}_{i j}^{m}=\{c \mid c \in[j-m, j], c \neq j-m+i, c \in \mathbb{Z}\} .
\end{aligned}
$$

For any given positive integer $s$ with $s<m$, we introduce the following notations:

- $\mu_{j, i}^{m, s}$ is the sum of products of all the different combinations of $m-s$ elements in $\mathcal{A}_{i j}$;

- $\nu_{j, i}^{m, s}$ is the sum of products of all the different combinations of $m-s$ elements in $\mathcal{B}_{i j}$;

- $\sigma_{j, i}^{m, s}$ is the sum of products of all the different combinations of $m-s$ elements in $\mathcal{C}_{i j}^{m}$.

When $s=m$, we set $\mu_{j, i}^{m, s}=\nu_{j, i}^{m, s}=\sigma_{j, i}^{m, s}=1$ for all $i, j$.

In order to construct a class of numerical methods for (1.1) in the subsequent section, we first consider an approximation to the Caputo derivative at $t_{n}$. The approximation is divided into the following two cases: 
(i) When $t \in\left[t_{j-m}, t_{j}\right](m<j \leq n, m<n \leq N), y(t)$ can be approximated by the Lagrange interpolation polynomial of degree $m$ :

$$
L_{m, j}(t)=\sum_{i=0}^{m} y\left(t_{j-i}\right) \prod_{l=0, l \neq i}^{m} \frac{t-t_{j-l}}{t_{j-i}-t_{j-l}},
$$

and thus

$$
\begin{aligned}
& \frac{1}{\Gamma(1-\gamma)} \int_{t_{j-m}}^{t_{j}} \frac{y^{\prime}(v)}{\left(t_{n}-v\right)^{\gamma}} d v \approx \frac{1}{\Gamma(1-\gamma)} \int_{t_{j-m}}^{t_{j}} \frac{L_{m, j}^{\prime}(v)}{\left(t_{n}-v\right)^{\gamma}} d v \\
= & \frac{1}{\Gamma(1-\gamma)} \sum_{i=0}^{m} \frac{(-1)^{i} y\left(t_{j-i}\right)}{i !(m-i) ! h^{m}} \int_{t_{j-m}}^{t_{j}}\left(t_{n}-v\right)^{-\gamma}\left[\prod_{l=0, l \neq i}^{m}\left(v-t_{j-l}\right)\right]^{\prime} d v \\
= & \left.\frac{1}{\Gamma(1-\gamma)}\left\{\sum_{i=0}^{m} \frac{(-1)^{i+1} y\left(t_{j-i}\right)}{i !(m-i) ! h^{m}}\left[\sum_{s=1}^{m} \frac{\left(t_{n}-v\right)^{s-\gamma}}{\prod_{l=1}^{s}(l-\gamma)}\left(\prod_{l=0, l \neq i}^{m}\left(v-t_{j-l}\right)\right)^{(s)}\right]\right\}\right|_{t_{j-m}} ^{t_{j}} \\
= & \frac{h^{-\gamma}}{\Gamma(1-\gamma)} \sum_{i=0}^{m} \omega_{i, j, n}^{m} y\left(t_{j-i}\right),
\end{aligned}
$$

where

$$
\omega_{i, j, n}^{m}=\frac{(-1)^{i+1}}{i !(m-i) !} \sum_{s=1}^{m}\left[\frac{s !}{\prod_{l=1}^{s}(l-\gamma)}\left(\mu_{m, i}^{m, s}(n-j)^{s-\gamma}-\nu_{m, i}^{m, s}(n-j+m)^{s-\gamma}\right)\right], \quad 0 \leq i \leq m .
$$

(ii) When $t \in\left[t_{0}, t_{j}\right](1 \leq j \leq m, j \leq n \leq N), y(t)$ can be approximated by $L_{m, m}(t)$ and thus

$$
\frac{1}{\Gamma(1-\gamma)} \int_{t_{0}}^{t_{j}} \frac{y^{\prime}(v)}{\left(t_{n}-v\right)^{\gamma}} d v \approx \frac{h^{-\gamma}}{\Gamma(1-\gamma)} \sum_{i=0}^{m} \varpi_{i, j, n}^{m} y\left(t_{m-i}\right)
$$

where

$$
\varpi_{i, j, n}^{m}=\frac{(-1)^{i+1}}{i !(m-i) !} \sum_{s=1}^{m}\left[\frac{s !}{\prod_{l=1}^{s}(l-\gamma)}\left(\sigma_{j, i}^{m, s}(n-j)^{s-\gamma}-\nu_{m, i}^{m, s} n^{s-\gamma}\right)\right], \quad 0 \leq i \leq m .
$$

Let $R_{m}^{n}$ be the truncation error of Lagrange interpolation for the Caputo derivative at $t_{n}$. Then, when $1 \leq n \leq m$, we have that

$$
\begin{aligned}
{ }_{t_{0}}^{C} D_{t}^{\gamma} y\left(t_{n}\right) & =\frac{1}{\Gamma(1-\gamma)} \int_{t_{0}}^{t_{n}} \frac{y^{\prime}(v)}{\left(t_{n}-v\right)^{\gamma}} d v=\frac{1}{\Gamma(1-\gamma)} \int_{t_{0}}^{t_{n}} \frac{L_{m, m}^{\prime}(v)}{\left(t_{n}-v\right)^{\gamma}} d v+R_{m}^{n} \\
& =\frac{h^{-\gamma}}{\Gamma(1-\gamma)} \sum_{i=0}^{m} \varpi_{m-i, n, n}^{m} y\left(t_{i}\right)+R_{m}^{n},
\end{aligned}
$$

and, when $m<n \leq N$, we have by selecting a positive integer $r$ with $m(r+1) \geq n$ that

$$
{ }_{t_{0}}^{C} D_{t}^{\gamma} y\left(t_{n}\right)=\frac{1}{\Gamma(1-\gamma)} \int_{t_{0}}^{t_{n}} \frac{y^{\prime}(v)}{\left(t_{n}-v\right)^{\gamma}} d v
$$




$$
\begin{aligned}
& =\frac{1}{\Gamma(1-\gamma)} \int_{t_{0}}^{t_{n-r m}} \frac{L_{m, m}^{\prime}(v)}{\left(t_{n}-v\right)^{\gamma}} d v+\frac{1}{\Gamma(1-\gamma)} \sum_{j=0}^{r-1} \int_{t_{n-(r-j) m}}^{t_{n-(r-j-1) m}} \frac{L_{m, n-(r-j-1) m}^{\prime}(v)}{\left(t_{n}-v\right)^{\gamma}} d v+R_{m}^{n} \\
& =\frac{h^{-\gamma}}{\Gamma(1-\gamma)} \Lambda_{n, m}^{n} \Omega_{n, m} \mathcal{Y}_{n}+R_{m}^{n}
\end{aligned}
$$

where $\mathcal{Y}_{n}=\left(y\left(t_{0}\right), y\left(t_{1}\right), \cdots, y\left(t_{n}\right)\right)^{T}$ and

$$
\Omega_{n, m}=\left[\begin{array}{ccccccc}
\varpi_{m, 1, n}^{m} & \cdots & \cdots & \varpi_{0,1, n}^{m} & 0 & \cdots & 0 \\
\vdots & \cdots & \cdots & \vdots & \vdots & \cdots & \vdots \\
\varpi_{m, m-1, n}^{m} & \cdots & \cdots & \varpi_{0, m-1, n}^{m} & 0 & \cdots & 0 \\
\varpi_{m, m, n}^{m} & \cdots & \cdots & \varpi_{0, m, n}^{m} & 0 & \cdots & 0 \\
0 & \omega_{m, m+1, n}^{m} & \cdots & \cdots & \omega_{0, m+1, n}^{m} & \ddots & \vdots \\
\vdots & \ddots & \ddots & & & \ddots & 0 \\
0 & \cdots & 0 & \omega_{m, n, n}^{m} & \cdots & \cdots & \omega_{0, n, n}^{m}
\end{array}\right]_{n \times(n+1)}
$$

and $\Lambda_{n, m}^{n}$ denotes the $n$-th row of matrix

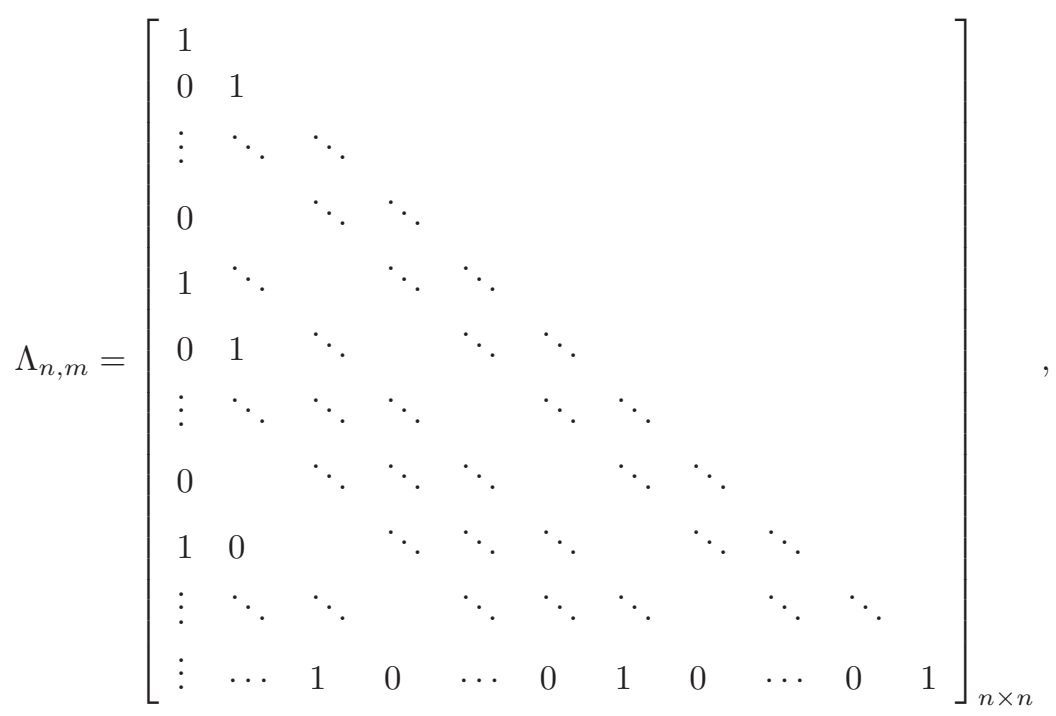

in which the first column of $\Lambda_{n, m}$ is the vector $(1, \overbrace{0, \cdots, 0}^{m-1}, 1, \overbrace{0, \cdots, 0}^{m-1}, 1, \cdots)^{T}$. Write

$$
\theta_{i, n}^{m}=\left\{\begin{array}{l}
\varpi_{m-i, n, n}^{m}, \quad 0 \leq i \leq m, 1 \leq n \leq m, \\
(i+1) \text {-th element of vector } \Lambda_{n, m}^{n} \Omega_{n, m}, \quad 0 \leq i \leq n, m<n \leq N .
\end{array}\right.
$$

Then Eqs. (2.1) and (2.2) can be rewritten as

$$
{ }_{t_{0}}^{C} D_{t}^{\gamma} y\left(t_{n}\right)= \begin{cases}\frac{h^{-\gamma}}{\Gamma(1-\gamma)} \sum_{i=0}^{m} \theta_{i, n}^{m} y\left(t_{i}\right)+R_{m}^{n}, & 1 \leq n \leq m, \\ \frac{h^{-\gamma}}{\Gamma(1-\gamma)} \sum_{i=0}^{n} \theta_{i, n}^{m} y\left(t_{i}\right)+R_{m}^{n}, & m<n \leq N .\end{cases}
$$

In order to analyze truncation error $R_{m}^{n}$ in $(2.3)$, the following lemma is needed. 
Lemma 2.1 (see e.g. [35]). Suppose that $y(t) \in C^{(m+1)}\left(\left[t_{0}, T\right]\right)$. Then the interpolation $L_{m, j}(t)$ satisfies for all $t \in\left(t_{j-m}, t_{j}\right)(m \leq j \leq N)$ that

$$
\begin{aligned}
& \left\|y(t)-L_{m, j}(t)\right\|_{\infty} \leq M_{m+1}^{j} h^{m+1}, \\
& \left\|y^{\prime}(t)-L_{m, j}^{\prime}(t)\right\|_{\infty} \leq M_{m+1}^{j} h^{m},
\end{aligned}
$$

where $M_{m+1}^{j}=\max _{t \in\left[t_{j-m}, t_{j}\right]}\left\|y^{(m+1)}(t)\right\|_{\infty}$.

We are now in a position to give the estimation of the truncation error $R_{m}^{n}$ in (2.3).

Theorem 2.1. Let $y(t) \in C^{(m+1)}\left(\left[t_{0}, T\right]\right)$. Then, there exists a constant $c_{0}>0$ such that

$$
\left\|R_{m}^{n}\right\|_{\infty} \leq c_{0} h^{m-\gamma+1}, \quad 1 \leq n \leq N, \quad 0<\gamma<1 .
$$

Proof. In the following, we will perform the proof in two cases. When $1 \leq n \leq m$, it follows from (2.1) and Lemma 2.1 that

$$
\begin{aligned}
& \left\|R_{m}^{n}\right\|_{\infty}=\left\|\frac{1}{\Gamma(1-\gamma)}\left[\int_{t_{0}}^{t_{n}} \frac{y^{\prime}(v)}{\left(t_{n}-v\right)^{\gamma}} d v-\int_{t_{0}}^{t_{n}} \frac{L_{m, m}^{\prime}(v)}{\left(t_{n}-v\right)^{\gamma}} d v\right]\right\|_{\infty} \\
= & \left\|\frac{1}{\Gamma(1-\gamma)}\left[\left.\frac{y(v)-L_{m, m}(v)}{\left(t_{n}-v\right)^{\gamma}}\right|_{t_{0}} ^{t_{n-1}}-\gamma \int_{t_{0}}^{t_{n-1}} \frac{y(v)-L_{m, m}(v)}{\left(t_{n}-v\right)^{\gamma+1}} d v+\int_{t_{n-1}}^{t_{n}} \frac{\left[y(v)-L_{m, m}(v)\right]^{\prime}}{\left(t_{n}-v\right)^{\gamma}} d v\right]\right\|_{\infty} \\
= & \left\|\frac{-\gamma}{\Gamma(1-\gamma)} \int_{t_{0}}^{t_{n-1}} \frac{y(v)-L_{m, m}(v)}{\left(t_{n}-v\right)^{\gamma+1}} d v+\frac{1}{\Gamma(1-\gamma)} \int_{t_{n-1}}^{t_{n}} \frac{\left[y(v)-L_{m, m}(v)\right]^{\prime}}{\left(t_{n}-v\right)^{\gamma}} d v\right\|_{\infty} \\
\leq & \frac{\gamma M_{m+1}^{m}}{\Gamma(1-\gamma)} h^{m+1}\left|\int_{t_{0}}^{t_{n-1}}\left(t_{n}-v\right)^{-\gamma-1} d v\right|+\frac{M_{m+1}^{m}}{\Gamma(1-\gamma)} h^{m}\left|\int_{t_{n-1}}^{t_{n}}\left(t_{n}-v\right)^{-\gamma} d v\right| \\
\leq & \frac{M_{m+1}^{m}}{\Gamma(1-\gamma)}\left(1+\frac{1}{1-\gamma}\right) h^{m-\gamma+1} .
\end{aligned}
$$

When $m<n \leq N$, by $(2.2)$ and Lemma 2.1 we have that

$$
\begin{aligned}
& \left\|R_{m}^{n}\right\|_{\infty}=\left\|\frac{1}{\Gamma(1-\gamma)}\left[\int_{t_{0}}^{t_{n}} \frac{y^{\prime}(v)}{\left(t_{n}-v\right)^{\gamma}} d v-\int_{t_{0}}^{t_{n-r m}} \frac{L_{m, m}^{\prime}(v)}{\left(t_{n}-v\right)^{\gamma}} d v-\sum_{j=0}^{r-1} \int_{t_{n-(r-j) m}}^{t_{n-(r-j-1) m}} \frac{L_{m, n-(r-j-1) m}^{\prime}(v)}{\left(t_{n}-v\right)^{\gamma}} d v\right]\right\|_{\infty} \\
& =\| \frac{1}{\Gamma(1-\gamma)}\left[\left.\frac{y(v)-L_{m, m}(v)}{\left(t_{n}-v\right)^{\gamma}}\right|_{t_{0}} ^{t_{n-r m}}-\gamma \int_{t_{0}}^{t_{n-r m}} \frac{y(v)-L_{m, m}(v)}{\left(t_{n}-v\right)^{\gamma+1}} d v+\left.\sum_{j=0}^{r-2} \frac{y(v)-L_{m, n-(r-j-1) m}(v)}{\left(t_{n}-v\right)^{\gamma}}\right|_{t_{n-(r-j) m}} ^{t_{n-(n-j-1) m}}\right. \\
& \left.\quad-\gamma \sum_{j=0}^{r-2} \int_{t_{n-(r-j) m}}^{t_{n-(r-j-1) m}} \frac{y(v)-L_{m, n-(r-j-1) m}(v)}{\left(t_{n}-v\right)^{\gamma+1}} d v+\int_{t_{n-m}}^{t_{n}} \frac{\left[y(v)-L_{m, n}(v)\right]^{\prime}}{\left(t_{n}-v\right)^{\gamma}} d v\right] \|_{\infty} \\
& =\| \frac{-\gamma}{\Gamma(1-\gamma)} \int_{t_{0}}^{t_{n-r m}} \frac{y(v)-L_{m, m}(v)}{\left(t_{n}-v\right)^{\gamma+1}} d v+\frac{-\gamma}{\Gamma(1-\gamma)} \sum_{j=0}^{r-2} \int_{t_{n-(r-j) m}}^{t_{n-(r-j-1) m}} \frac{y(v)-L_{m, n-(r-j-1) m}(v)}{\left(t_{n}-v\right)^{\gamma+1}} d v \\
& \quad+\frac{1}{\Gamma(1-\gamma)} \int_{t_{n-m}}^{t_{n}} \frac{\left[y(v)-L_{m, n}(v)\right]^{\prime}}{\left(t_{n}-v\right)^{\gamma}} d v \|_{\infty} \frac{\gamma M_{m+1}^{m}}{\Gamma(1-\gamma)} h^{m+1}\left|\int_{t_{0}}^{t_{n-r m}}\left(t_{n}-v\right)^{-\gamma-1} d v\right| \\
& \quad+\frac{\gamma}{\Gamma(1-\gamma)} h^{m+1}\left|\sum_{j=0}^{r-2} M_{m+1}^{n-(r-j-1) m} \int_{t_{n-(n-j) m}}^{t_{n-(r-j-1) m}}\left(t_{n}-v\right)^{-\gamma-1} d v\right|+\frac{M_{m+1}^{n}}{\Gamma(1-\gamma)} h^{m}\left|\int_{t_{n-m}}^{t_{n}}\left(t_{n}-v\right)^{-\gamma} d v\right|
\end{aligned}
$$


Table 2.1: Absolute errors and convergence orders of schemes (2.3) for computing ${ }_{0}^{C} D_{t}^{\gamma}[\sin (\pi t)]$.

\begin{tabular}{|c|c|c|c|c|c|c|c|}
\hline \multirow[b]{2}{*}{$\gamma$} & \multirow[b]{2}{*}{$h$} & \multicolumn{2}{|c|}{$m=1$} & \multicolumn{2}{|c|}{$m=2$} & \multicolumn{2}{|c|}{$m=3$} \\
\hline & & $\operatorname{err}_{1}(h)$ & $\bar{p}_{1}$ & $\operatorname{err}_{2}(h)$ & $\bar{p}_{2}$ & $\operatorname{err}_{3}(h)$ & $\bar{p}_{3}$ \\
\hline \multirow[t]{4}{*}{0.25} & $1 / 4$ & $7.0241 \mathrm{e}-2$ & - & $2.6755 \mathrm{e}-2$ & - & $1.5071 \mathrm{e}-2$ & - \\
\hline & $1 / 8$ & $2.4071 \mathrm{e}-2$ & 1.5450 & $4.1355 \mathrm{e}-3$ & 2.6937 & $1.1982 \mathrm{e}-3$ & 3.6528 \\
\hline & $1 / 16$ & $7.7592 \mathrm{e}-3$ & 1.6333 & $6.2175 \mathrm{e}-4$ & 2.7336 & $9.4202 \mathrm{e}^{-5}$ & 3.6690 \\
\hline & $1 / 32$ & $2.4595 \mathrm{e}-3$ & 1.6576 & $9.2843 \mathrm{e}-5$ & 2.7435 & $7.2358 \mathrm{e}-6$ & 3.7025 \\
\hline \multirow[t]{4}{*}{0.5} & $1 / 4$ & $2.2343 \mathrm{e}-1$ & - & $9.9015 \mathrm{e}-2$ & - & $5.5287 \mathrm{e}-2$ & - \\
\hline & $1 / 8$ & $8.8408 \mathrm{e}-2$ & 1.3375 & $1.8250 \mathrm{e}-2$ & 2.4397 & $5.2092 \mathrm{e}-3$ & 3.4078 \\
\hline & $1 / 16$ & $3.2846 \mathrm{e}-2$ & 1.4285 & $3.2643 \mathrm{e}-3$ & 2.4831 & $4.7478 \mathrm{e}-4$ & 3.4557 \\
\hline & $1 / 32$ & $1.1950 \mathrm{e}-2$ & 1.4588 & $5.7926 \mathrm{e}-4$ & 2.4945 & $4.2526 \mathrm{e}-5$ & 3.4809 \\
\hline \multirow[t]{4}{*}{0.75} & $1 / 4$ & $5.5005 \mathrm{e}-1$ & - & $2.6502 \mathrm{e}-1$ & - & $1.4931 \mathrm{e}-1$ & - \\
\hline & $1 / 8$ & $2.4642 \mathrm{e}-1$ & 1.1585 & $5.8219 \mathrm{e}-2$ & 2.1865 & $1.6704 \mathrm{e}-2$ & 3.1600 \\
\hline & $1 / 16$ & $1.0622 \mathrm{e}-1$ & 1.2140 & $1.2381 \mathrm{e}-2$ & 2.2334 & $1.7908 \mathrm{e}-3$ & 3.2216 \\
\hline & $1 / 32$ & $4.5201 \mathrm{e}-2$ & 1.2327 & $2.6109 \mathrm{e}-3$ & 2.2455 & $1.8939 \mathrm{e}-4$ & 3.2411 \\
\hline
\end{tabular}

$\leq \frac{M_{m+1}}{\Gamma(1-\gamma)}\left[\frac{1}{(r m)^{\gamma}}+\frac{1}{m^{\gamma}}+\frac{1}{(1-\gamma) m^{\gamma-1}}\right] h^{m-\gamma+1}$,

where $M_{m+1}=\max _{t \in\left[t_{0}, T\right]}\left\|y^{(m+1)}(t)\right\|_{\infty}$. Therefore, this completes the proof.

We have noted that Li et al. [22] also used the $m$-th Lagrange interpolation to approximate the $\gamma$-order Caputo dervative. When $m=1$, their scheme is the same as scheme (2.3), which is just $L 1$ method (see e.g. [30,36]). However, when $m>1$, they use $L_{j, j}(t)$ to approximate $y(t)$ on $\in\left[t_{j-1}, t_{j}\right](0 \leq j \leq m)$, which leads to that the approximation can not arrive at the accuracy of order $m-\gamma+1$ on $\left[t_{0}, t_{j}\right]$ unless $y^{(j)}\left(t_{0}\right)=0(0 \leq j \leq m)$. While, in our approach, $y(t)$ is approximated by the $m$-th interpolation $L_{m, m}(t)$ on $\left[t_{0}, t_{j}\right](1 \leq j \leq m)$, which, together with Theorem 2.1, implies a high-accuracy numerical approximation for $y(t)$ can be achieved. This also can be testified by the following numerical example.

Example 2.1. In the following, we use the above two numerical schemes to compute the $\gamma$ order Caputo derivative of function $y(t)=\sin (\pi t)$ on $[0,5]$. Let

$$
\operatorname{err}_{m}(h)=\max _{1 \leq n \leq N}\left|R_{m}^{n}\right|, \quad \bar{p}_{m}=\log _{2}\left[\frac{\operatorname{err}_{m}(h)}{\operatorname{err}_{m}(h / 2)}\right]
$$

to characterize the absolute errors and convergence orders of the schemes, respectively. Taking $h=1 / 2^{i}(i=2,3,4,5)$ and $m=1,2,3$, respectively, and then applying scheme (2.3) and $\mathrm{Li}$ et al.'s scheme $(7)$ in $[22]$ to compute ${ }_{0}^{C} D_{t}^{\gamma}[\sin (\pi t)](\gamma=0.25,0.5,0.75)$ on $[0,5]$. The derived numerical results are displayed in Tables 2.1-2.2, which show that, when $m=1$, the both schemes have the same accuracy under the same stepsize; and when $m>1$, scheme (2.3) can arrive at the convergence order $m-\gamma+1$, but Li et al's scheme doesn't arrive at the desired order $m-\gamma+1$.

\section{The Extended BVMs for CFDEs}

In the recent years, BVMs have been used successfully to solve various differential equations (see e.g. [1, 2, 4-6, 10-12, 15, 16, 18, 19,38,39,41-43]). A detailed introduction on BVMs for 
Table 2.2: Absolute errors and convergence orders of Li et al's scheme for computing ${ }_{0}^{C} D_{t}^{\gamma}[\sin (\pi t)]$.

\begin{tabular}{|c|c|c|c|c|c|c|c|}
\hline \multirow[b]{2}{*}{$\gamma$} & \multirow[b]{2}{*}{$h$} & \multicolumn{2}{|c|}{$m=1$} & \multicolumn{2}{|c|}{$m=2$} & \multicolumn{2}{|c|}{$m=3$} \\
\hline & & $\operatorname{err}_{1}(h)$ & $\bar{p}_{1}$ & $\operatorname{err}_{2}(h)$ & $\bar{p}_{2}$ & $\operatorname{err}_{3}(h)$ & $\bar{p}_{3}$ \\
\hline \multirow[t]{4}{*}{0.25} & $1 / 4$ & $7.0241 \mathrm{e}-2$ & - & $3.0561 \mathrm{e}-2$ & - & $2.9155 \mathrm{e}-2$ & - \\
\hline & $1 / 8$ & $2.4071 \mathrm{e}-2$ & 1.5450 & $5.1022 \mathrm{e}-3$ & 2.5825 & $4.5778 \mathrm{e}^{-}-3$ & 2.6710 \\
\hline & $1 / 16$ & $7.7592 \mathrm{e}-3$ & 1.6333 & $8.2352 \mathrm{e}-4$ & 2.6312 & $7.3963 \mathrm{e}-4$ & 2.6298 \\
\hline & $1 / 32$ & $2.4595 \mathrm{e}-3$ & 1.6576 & $1.3124 \mathrm{e}-4$ & 2.6496 & $1.1220 \mathrm{e}-4$ & 2.7208 \\
\hline \multirow[t]{4}{*}{0.5} & $1 / 4$ & $2.2343 \mathrm{e}-1$ & - & $1.0680 \mathrm{e}-1$ & - & $1.0365 \mathrm{e}-1$ & - \\
\hline & $1 / 8$ & $8.8408 \mathrm{e}-2$ & 1.3375 & $2.0414 \mathrm{e}-2$ & 2.3873 & $1.9073 \mathrm{e}^{-2}$ & 2.4421 \\
\hline & $1 / 16$ & $3.2846 \mathrm{e}-2$ & 1.4285 & $3.7627 \mathrm{e}-3$ & 2.4397 & $3.5217 \mathrm{e}-3$ & 2.4372 \\
\hline & $1 / 32$ & $1.1950 \mathrm{e}-2$ & 1.4588 & $6.8363 \mathrm{e}-4$ & 2.4605 & $6.3636 \mathrm{e}-4$ & 2.4684 \\
\hline \multirow[t]{4}{*}{0.75} & $1 / 4$ & $5.5005 \mathrm{e}-1$ & - & $2.7312 \mathrm{e}-1$ & - & $2.6966 \mathrm{e}-1$ & - \\
\hline & $1 / 8$ & $2.4642 \mathrm{e}-1$ & 1.1585 & $6.0728 \mathrm{e}-2$ & 2.1691 & $5.9183 \mathrm{e}-2$ & 2.1879 \\
\hline & $1 / 16$ & $1.0622 \mathrm{e}-1$ & 1.2140 & $1.3031 \mathrm{e}-2$ & 2.2204 & $1.2575 \mathrm{e}-2$ & 2.2346 \\
\hline & $1 / 32$ & $4.5201 \mathrm{e}-2$ & 1.2327 & $2.7655 \mathrm{e}^{-}-3$ & 2.2363 & $2.6915 \mathrm{e}^{-3}$ & 2.2241 \\
\hline
\end{tabular}

ordinary differential equations (ODEs) refers to Brugnano and Trigiante's monograph [4], where BVMs are verified to have the better stability than the classical linear multistep methods. We note that, up to now, no result has been found on BVMs for CFDEs (1.1). Hence, in this section, we will extend BVMs to solve (1.1).

For convenience, we first give a brief review to the BVMs for the following $d$-dimensional problems of ODEs:

$$
y^{\prime}(t)=f(t, y(t)), \quad t \in\left[t_{0}, T\right] ; \quad y\left(t_{0}\right)=y_{0} .
$$

For solving (3.1), the BVMs with $k_{1}$ initial values $\left\{y_{i}\right\}_{i=0}^{k_{1}-1}$ and $k_{2}\left(=k-k_{1}\right)$ final values $\left\{y_{i}\right\}_{i=N-k_{2}+1}^{N}(N \in \mathbb{N})$ can be defined as follows:

$$
\begin{array}{ll}
\sum_{i=-k_{1}}^{k_{2}} \alpha_{i+k_{1}} y_{n+i}=h \sum_{i=-k_{1}}^{k_{2}} \beta_{i+k_{1}} f_{n+i}, & n=k_{1}, k_{1}+1 \ldots, N-k_{2}, \\
\sum_{i=0}^{k} \alpha_{i}^{(j)} y_{i}=h \sum_{i=0}^{k} \beta_{i}^{(j)} f_{i}, & j=1,2, \ldots, k_{1}-1, \\
\sum_{i=0}^{k} \alpha_{k-i}^{(j)} y_{N-i}=h \sum_{i=0}^{k} \beta_{k-i}^{(j)} f_{N-i}, & j=N-k_{2}+1, N-k_{2}+2, \ldots, N,
\end{array}
$$

where $y_{n} \approx y\left(t_{n}\right), f_{n}=f\left(t_{n}, y_{n}\right)$, and $\alpha_{i}, \beta_{i}, \alpha_{i}^{(j)}$ and $\beta_{i}^{(j)}$ are some real coefficients such that schemes (3.2)-(3.4) have the same consistency order. Let $\otimes$ be the Kronecker product, $I_{d}$ the 
$d \times d$ identity matrix, $Y=\left(y_{1}^{T}, y_{2}^{T}, \ldots, y_{N}^{T}\right)^{T}, F(Y)=\left(f_{1}^{T}, f_{2}^{T}, \ldots, f_{N}^{T}\right)^{T}$,

$$
A^{e}:=\left[a_{0} \mid A\right]=\left[\begin{array}{c|cccccc}
\alpha_{0}^{(1)} & \alpha_{1}^{(1)} & \cdots & \alpha_{k}^{(1)} & & & \\
\vdots & \vdots & \cdots & \vdots & & & \\
\alpha_{0}^{\left(k_{1}-1\right)} & \alpha_{1}^{\left(k_{1}-1\right)} & \cdots & \alpha_{k}^{\left(k_{1}-1\right)} & & & \\
\alpha_{0} & \alpha_{1} & \cdots & \alpha_{k} & & & \\
& \alpha_{0} & \alpha_{1} & \cdots & \alpha_{k} & & \\
& & \ddots & \ddots & \ddots & \ddots & \\
& & & \alpha_{0} & \alpha_{1} & \cdots & \alpha_{k} \\
& & & \alpha_{0}^{\left(N-k_{2}+1\right)} & \alpha_{1}^{\left(N-k_{2}+1\right)} & \cdots & \alpha_{k}^{\left(N-k_{2}+1\right)} \\
& & \vdots & \vdots & \cdots & \vdots \\
& & & \alpha_{0}^{(N)} & \alpha_{1}^{(N)} & \cdots & \alpha_{k}^{(N)}
\end{array}\right] \in \mathbb{R}^{N \times(N+1)}
$$

and $B^{e}:=\left[b_{0} \mid B\right]$, which is defined similar to $A^{e}$ by replacing $\alpha_{i}$ (resp. $\alpha_{i}^{(j)}$ ) with $\beta_{i}$ (resp. $\left.\beta_{i}^{(j)}\right)$. Then BVMs (3.2)-(3.4) can be written in a compact form:

$$
\left(A \otimes I_{d}\right) Y+a_{0} \otimes y_{0}=h\left(B \otimes I_{d}\right) F(Y)+h b_{0} \otimes f\left(t_{0}, y_{0}\right) .
$$

A BVM (3.5) is called consistent of order $q$ if its local truncation error

$$
\check{\delta}:=\left(A \otimes I_{d}\right) \bar{Y}+a_{0} \otimes y_{0}-h\left(B \otimes I_{d}\right) F(\bar{Y})-h b_{0} \otimes f\left(t_{0}, y_{0}\right)=\mathcal{O}\left(h^{q+1}\right),
$$

where $\bar{Y}=\left(y\left(t_{1}\right)^{T}, \ldots, y\left(t_{N}\right)^{T}\right)^{T}$. Write $Z=\left(z_{1}^{T}, \ldots, z_{N}^{T}\right)^{T}$, where

$$
z_{n}=\left\{\begin{array}{cc}
\frac{h^{-\gamma}}{\Gamma(1-\gamma)} \sum_{i=0}^{m} \theta_{i, n}^{m} y_{i}, & 1 \leq n \leq m, \\
\frac{h^{-\gamma}}{\Gamma(1-\gamma)} \sum_{i=0}^{n} \theta_{i, n}^{m} y_{i}, & m<n \leq N
\end{array}\right.
$$

denotes the interpolation approximation for $z\left(t_{n}\right):={ }_{t_{0}}^{C} D_{t}^{\gamma} y\left(t_{n}\right), \mathbf{0}=(0, \ldots, 0)^{T} \in \mathbb{R}^{d}$ and $F(Y, Z)=\left(f\left(t_{1}, y_{1}, z_{1}\right)^{T}, \ldots, f\left(t_{N}, y_{N}, z_{N}\right)^{T}\right)^{T}$. Adapting BVMs (3.5) to problem (1.1) yields the following extended BVMs:

$$
\left(A \otimes I_{d}\right) Y+a_{0} \otimes y_{0}=h\left(B \otimes I_{d}\right) F(Y, Z)+h b_{0} \otimes f\left(t_{0}, y_{0}, \mathbf{0}\right) .
$$

Let $\bar{Z}=\left(z\left(t_{1}\right)^{T}, \ldots, z\left(t_{N}\right)^{T}\right)^{T}$. An extended BVM (3.8) is called consistent of order $q$ if its local truncation error

$$
\tilde{\delta}:=\left(A \otimes I_{d}\right) \bar{Y}+a_{0} \otimes y\left(t_{0}\right)-h\left(B \otimes I_{d}\right) F(\bar{Y}, \bar{Z})-h b_{0} \otimes f\left(t_{0}, y\left(t_{0}\right), \mathbf{0}\right)=\mathcal{O}\left(h^{q+1}\right) .
$$

Remark 3.1. Since, for problem (3.1) it holds that

$$
F(\bar{Y})=\left(\left[y^{\prime}\left(t_{1}\right)\right]^{T}, \ldots,\left[y^{\prime}\left(t_{N}\right)\right]^{T}\right)^{T}, \quad f\left(t_{0}, y_{0}\right)=y^{\prime}\left(t_{0}\right),
$$

and for problem (1.1) it holds that

$$
F(\bar{Y}, \bar{Z})=\left(\left[y^{\prime}\left(t_{1}\right)\right]^{T}, \ldots,\left[y^{\prime}\left(t_{N}\right)\right]^{T}\right)^{T}, \quad f\left(t_{0}, y\left(t_{0}\right), \mathbf{0}\right)=y^{\prime}\left(t_{0}\right),
$$

we conclude from (3.6) and (3.9) that an extended BVM (3.8) and its corresponding underlying BVM (3.5) have the same consistency order. 


\section{Local Stability and Unique Solvability of the Extended BVMs}

Let $\langle\cdot, \cdot\rangle$ be the Euclidean inner product on $\mathbb{R}^{d}$ and $\|\cdot\|_{2}$ the induced norm by this inner product. For any given vectors $U=\left(u_{1}^{T}, \ldots, u_{N}^{T}\right)^{T}$ and $V=\left(v_{1}^{T}, \ldots, v_{N}^{T}\right)^{T}$ in $\mathbb{R}^{N d}$, we further define the inner product $\langle\cdot, \cdot\rangle_{h}$ and the corresponding norm $\|\cdot\|_{h}$ as follows:

$$
\langle U, V\rangle_{h}=h \sum_{i=1}^{N}\left\langle u_{i}, v_{i}\right\rangle, \quad\|U\|_{h}=\sqrt{h \sum_{i=1}^{N}\left\|u_{i}\right\|_{2}^{2}} .
$$

Based on vector norm $\|\cdot\|_{h}$, we also introduce the following matrix norm $\|\cdot\|_{h}$ :

$$
\|\mathcal{M}\|_{h}=\max _{\|U\|_{h}=1}\|\mathcal{M} U\|_{h}, \quad \forall \mathcal{M} \in \mathbb{R}^{(N d) \times(N d)} .
$$

It is easy to check that $\|\mathcal{M}\|_{h}=\|\mathcal{M}\|_{2}$ for all $\mathcal{M} \in \mathbb{R}^{(N d) \times(N d)}$. In the following, we always assume that there exist constants $L_{1}, L_{2}>0$ such that function $f$ in (1.1) satisfies that

$$
\begin{aligned}
& \langle f(t, y, z)-f(t, \hat{y}, z), y-\hat{y}\rangle \leq L_{1}\|y-\hat{y}\|_{2}^{2}, \quad \forall t \in\left[t_{0}, T\right], y, \hat{y}, z \in \mathbb{R}^{d}, \\
& \|f(t, y, z)-f(t, y, \hat{z})\|_{2} \leq L_{2}\|z-\hat{z}\|_{2}, \quad \forall t \in\left[t_{0}, T\right], y, z, \hat{z} \in \mathbb{R}^{d} .
\end{aligned}
$$

On the basis of the above settings, we will deal with local stability and unique solvability of the extended BVMs (3.8). Let $\hat{Y}=\left(\hat{y}_{1}^{T}, \ldots, \hat{y}_{N}^{T}\right)^{T}$ be the solution of the following perturbed equation with local perturbation $\delta \in \mathbb{R}^{N d}$ :

$$
\left(A \otimes I_{d}\right) \hat{Y}+a_{0} \otimes y_{0}=h\left(B \otimes I_{d}\right) F(\hat{Y}, \hat{Z})+h b_{0} \otimes f\left(t_{0}, y_{0}, \mathbf{0}\right)+\delta,
$$

where

$$
\hat{z}_{n}=\left\{\begin{array}{l}
\frac{h^{-\gamma}}{\Gamma(1-\gamma)} \sum_{i=0}^{m} \theta_{i, n}^{m} \hat{y}_{i}, \quad 1 \leq n \leq m, \\
\frac{h^{-\gamma}}{\Gamma(1-\gamma)} \sum_{i=0}^{n} \theta_{i, n}^{m} \hat{y}_{i}, \quad m<n \leq N,
\end{array} \quad \hat{y}_{0}=y_{0}, \quad \hat{Z}=\left(\hat{z}_{1}^{T}, \ldots, \hat{z}_{N}^{T}\right)^{T}\right.
$$

Subtracting (3.8) from (4.3) yields that

$$
\left(A \otimes I_{d}\right) \mathbf{v}=h\left(B \otimes I_{d}\right) \mathbf{w}+\delta,
$$

where $\mathbf{v}=\hat{Y}-Y, \mathbf{w}=F(\hat{Y}, \hat{Z})-F(Y, Z)$. An extended BVM (3.8) is called locally stable if there exists a constant $c>0$ such that $\|\mathbf{v}\|_{h} \leq c\|\delta\|_{h}$. In order to derive the local stability criterion, we first present the following lemma.

Lemma 4.1. Let $D=\operatorname{diag}\left(d_{1}, d_{2}, \ldots, d_{N}\right)$ be a given $N \times N$ positive diagonal matrix and conditions (4.1) and (4.2) hold. Then the vectors $\mathbf{v}$ and $\mathbf{w}$ in (4.4) satisfy that

$$
\left\langle\mathbf{v},\left(D \otimes I_{d}\right) \mathbf{w}\right\rangle_{h} \leq \mu\left\langle\mathbf{v},\left(D \otimes I_{d}\right) \mathbf{v}\right\rangle_{h},
$$

where

$$
\begin{aligned}
& \mu=L_{1}+\frac{L_{2} h^{-\gamma} \omega_{1} \omega_{2}}{\Gamma(1-\gamma)}, \quad \omega_{2}=\sqrt{\sum_{i=1}^{N} \sum_{j=1}^{N} \frac{d_{i}}{d_{j}}} \\
& \omega_{1}=\max \left\{\max _{0 \leq i \leq m} \max _{1 \leq n \leq m}\left|\theta_{i, n}^{m}\right|, \max _{0 \leq i \leq n} \max _{m<n \leq N}\left|\theta_{i, n}^{m}\right|\right\} .
\end{aligned}
$$


Proof. It follows from (4.1), (4.2) and the discrete Cauchy-Schwartz inequality that

$$
\begin{aligned}
& \left\langle\mathbf{v},\left(D \otimes I_{d}\right) \mathbf{w}\right\rangle_{h}=h \sum_{i=1}^{N} d_{i}\left\langle\hat{y}_{i}-y_{i}, f\left(t_{i}, \hat{y}_{i}, \hat{z}_{i}\right)-f\left(t_{i}, y_{i}, z_{i}\right)\right\rangle \\
= & h \sum_{i=1}^{N} d_{i}\left\langle\hat{y}_{i}-y_{i}, f\left(t_{i}, \hat{y}_{i}, \hat{z}_{i}\right)-f\left(t_{i}, y_{i}, \hat{z}_{i}\right)\right\rangle+h \sum_{i=1}^{N} d_{i}\left\langle\hat{y}_{i}-y_{i}, f\left(t_{i}, y_{i}, \hat{z}_{i}\right)-f\left(t_{i}, y_{i}, z_{i}\right)\right\rangle \\
\leq & L_{1} h \sum_{i=1}^{N} d_{i}\left\|\hat{y}_{i}-y_{i}\right\|_{2}^{2}+L_{2} h \sum_{i=1}^{N} d_{i}\left\|\hat{y}_{i}-y_{i}\right\|_{2}\left\|\hat{z}_{i}-z_{i}\right\|_{2} \\
\leq & L_{1}\left\langle\mathbf{v},\left(D \otimes I_{d}\right) \mathbf{v}\right\rangle_{h}+\frac{L_{2} h^{1-\gamma} \omega_{1}}{\Gamma(1-\gamma)} \sum_{i=1}^{N} d_{i}\left\|\hat{y}_{i}-y_{i}\right\|_{2} \sum_{j=1}^{N}\left\|\hat{y}_{j}-y_{j}\right\|_{2} \\
\leq & L_{1}\left\langle\mathbf{v},\left(D \otimes I_{d}\right) \mathbf{v}\right\rangle_{h}+\frac{L_{2} h^{1-\gamma} \omega_{1}}{\Gamma(1-\gamma)} \sqrt{\sum_{i=1}^{N} d_{i}} \sqrt{\sum_{i=1}^{N} d_{i}\left\|\hat{y}_{i}-y_{i}\right\|_{2}^{2}} \sqrt{\sum_{i=1}^{N} \frac{1}{d_{i}}} \sqrt{\sum_{i=1}^{N} d_{i}\left\|\hat{y}_{i}-y_{i}\right\|_{2}^{2}} \\
= & {\left[L_{1}+\frac{L_{2} h^{-\gamma} \omega_{1} \omega_{2}}{\Gamma(1-\gamma)}\right]\left\langle\mathbf{v},\left(D \otimes I_{d}\right) \mathbf{v}\right\rangle_{h} . }
\end{aligned}
$$

Hence the lemma is proven.

Refer to references $[10,15,37]$, we introduce the following hypothesis:

$\mathcal{H}$ : There exist $h_{0}>0, N \times N$ positive diagonal matrices $\tilde{D}$ and $\hat{D}$, and a positive bounded function $S(h)$ on $\left(0, h_{0}\right]$ such that

$$
\lambda_{\min }\left(\frac{\mathcal{A B}^{T}+\mathcal{B A}^{T}}{2}-h \mu \mathcal{B} \mathcal{B}^{T}\right) \geq S(h), \quad h \in\left(0, h_{0}\right],
$$

where $\mathcal{A}=(\tilde{D} A \hat{D}) \otimes I_{d}, \mathcal{B}=(\tilde{D} B \hat{D}) \otimes I_{d}$ and $\lambda_{\min }(\cdot)$ denotes the minimum eigenvalue of a matrix.

With the above arguments, a local stability criterion can be stated as follows.

Theorem 4.1. Assume that conditions (4.1), (4.2) and $\mathcal{H}$ are satisfied. Then the extended $B V M(3.8)$ is locally stable with

$$
\|\mathbf{v}\|_{h} \leq\left[\frac{\|\hat{D}\|_{h}\left\|\mathcal{B}^{T}\right\|_{h}\|\tilde{D}\|_{h}}{S(h)}\right]\|\delta\|_{h}, \quad h \in\left(0, h_{0}\right] .
$$

Proof. Since $S(h)$ is a positive bounded function on $\left(0, h_{0}\right]$, it suffices to prove that $(4.7)$ is true. Let $\tilde{\mathbf{v}}$ be a vector with $\mathcal{B}^{T} \tilde{\mathbf{v}}=\left(\hat{D}^{-1} \otimes I_{d}\right) \mathbf{v}$. Then equation (4.4) can be written as

$$
\mathcal{A B}^{T} \tilde{\mathbf{v}}=h \mathcal{B}\left(\hat{D}^{-1} \otimes I_{d}\right) \mathbf{w}+\left(\tilde{D} \otimes I_{d}\right) \delta .
$$

Taking the inner product with $\tilde{\mathbf{v}}$ on both sides of (4.8) yields that

$$
\left\langle\tilde{\mathbf{v}}, \mathcal{A B}^{T} \tilde{\mathbf{v}}\right\rangle_{h}=h\left\langle\tilde{\mathbf{v}}, \mathcal{B}\left(\hat{D}^{-1} \otimes I_{d}\right) \mathbf{w}\right\rangle_{h}+\left\langle\tilde{\mathbf{v}},\left(\tilde{D} \otimes I_{d}\right) \delta\right\rangle_{h} .
$$

In terms of the usual properties of inner product, (4.9) is equivalent to

$$
\left\langle\tilde{\mathbf{v}}, \mathcal{A B}^{T} \tilde{\mathbf{v}}\right\rangle_{h}=h\left\langle\mathbf{v},\left(\hat{D}^{-2} \otimes I_{d}\right) \mathbf{w}\right\rangle_{h}+\left\langle\tilde{\mathbf{v}},\left(\tilde{D} \otimes I_{d}\right) \delta\right\rangle_{h} .
$$


Also, it follows from Lemma 4.1 that

$$
\left\langle\mathbf{v},\left(\hat{D}^{-2} \otimes I_{d}\right) \mathbf{w}\right\rangle_{h} \leq \mu\left\langle\mathbf{v},\left(\hat{D}^{-2} \otimes I_{d}\right) \mathbf{v}\right\rangle_{h}=\mu\left\langle\tilde{\mathbf{v}}, \mathcal{B B}^{T} \tilde{\mathbf{v}}\right\rangle_{h}
$$

Substituting (4.11) into (4.10) and then applying the Cauchy-Schwartz inequality and equality: $\left\|\tilde{D} \otimes I_{d}\right\|_{h}=\|\tilde{D}\|_{h}$ derive that

$$
\left\langle\tilde{\mathbf{v}}, \mathcal{A B}^{T} \tilde{\mathbf{v}}\right\rangle_{h} \leq h \mu\left\langle\tilde{\mathbf{v}}, \mathcal{B B} \mathcal{B}^{T} \tilde{\mathbf{v}}\right\rangle_{h}+\left\langle\tilde{\mathbf{v}},\left(\tilde{D} \otimes I_{d}\right) \delta\right\rangle_{h} \leq h \mu\left\langle\tilde{\mathbf{v}}, \mathcal{B B} \mathcal{B}^{T} \tilde{\mathbf{v}}\right\rangle_{h}+\|\tilde{\mathbf{v}}\|_{h}\|\tilde{D}\|_{h}\|\delta\|_{h} .
$$

Moreover, applying identity: $\left\langle\tilde{\mathbf{v}}, \mathcal{A B}^{T} \tilde{\mathbf{v}}\right\rangle_{h}=\left\langle\tilde{\mathbf{v}},\left(\frac{\mathcal{A} \mathcal{B}^{T}+\mathcal{B} \mathcal{A}^{T}}{2}\right) \tilde{\mathbf{v}}\right\rangle_{h}$ to (4.12) gives that

$$
\left\langle\tilde{\mathbf{v}},\left(\frac{\mathcal{A B}^{T}+\mathcal{B} \mathcal{A}^{T}}{2}-h \mu \mathcal{B B} \mathcal{B}^{T}\right) \tilde{\mathbf{v}}\right\rangle_{h} \leq\|\tilde{\mathbf{v}}\|_{h}\|\tilde{D}\|_{h}\|\delta\|_{h}
$$

Whereas, by the property of inner product, the following inequality holds:

$$
\left\langle\tilde{\mathbf{v}},\left(\frac{\mathcal{A B}^{T}+\mathcal{B} \mathcal{A}^{T}}{2}-h \mu \mathcal{B B} \mathcal{B}^{T}\right) \tilde{\mathbf{v}}\right\rangle_{h} \geq \lambda_{\min }\left(\frac{\mathcal{A B}^{T}+\mathcal{B} \mathcal{A}^{T}}{2}-h \mu \mathcal{B B} \mathcal{B}^{T}\right)\|\tilde{\mathbf{v}}\|_{h}^{2} .
$$

A combination of (4.6), (4.13) and (4.14) generates that

$$
\|\tilde{\mathbf{v}}\|_{h} \leq \frac{\|\tilde{D}\|_{h}\|\delta\|_{h}}{S(h)}, \quad h \in\left(0, h_{0}\right] .
$$

Since $\mathbf{v}=\left(\hat{D} \otimes I_{d}\right) \mathcal{B}^{T} \tilde{\mathbf{v}}$, inequality (4.7) can be followed immediately by (4.15).

With Theorem 4.1, we can obtain a unique solvability criterion of the extended BVMs (3.8).

Theorem 4.2. Assume that conditions (4.1), (4.2) and $\mathcal{H}$ are satisfied. Then the extended $B V M(3.8)$ is uniquely solvable.

Proof. Write

$$
Q^{e}:=\left[q_{0} \mid Q\right]=\frac{h^{-\gamma}}{\Gamma(1-\gamma)}\left[\begin{array}{c|cccccc}
\theta_{0,1}^{m} & \theta_{1,1}^{m} & \cdots & \theta_{m, 1}^{m} & & & \\
\vdots & \vdots & \cdots & \vdots & & & \\
\theta_{0, m}^{m} & \theta_{1, m}^{m} & \cdots & \theta_{m, m}^{m} & & & \\
\theta_{0, m+1}^{m} & \theta_{1, m+1}^{m} & \cdots & \theta_{m, m+1}^{m} & \theta_{m+1, m+1}^{m} & & \\
\vdots & \vdots & \cdots & \vdots & \vdots & \ddots & \\
\theta_{0, N}^{m} & \theta_{1, N}^{m} & \cdots & \theta_{m, N}^{m} & \theta_{m+1, N}^{m} & \cdots & \theta_{N, N}^{m}
\end{array}\right] \in \mathbb{R}^{N \times(N+1)} .
$$

Then, it follows from (3.7) that $Z=q_{0} \otimes y_{0}+\left(Q \otimes I_{d}\right) Y$. Let $\breve{Y}$ be a vector such that $\mathcal{B}^{T} \breve{Y}=\left(\hat{D}^{-1} \otimes I_{d}\right) Y$. With this, the unique solvability of the extended BVM (3.8) is equivalent to the unique solvability of the following equation:

$$
\begin{gathered}
\mathcal{A B}^{T} \breve{Y}+\left(\tilde{D} a_{0}\right) \otimes y_{0}=h \mathcal{B}\left(\hat{D}^{-1} \otimes I_{d}\right) F\left(\left(\hat{D} \otimes I_{d}\right) \mathcal{B}^{T} \breve{Y}, q_{0} \otimes y_{0}+\left((Q \hat{D}) \otimes I_{d}\right) \mathcal{B}^{T} \breve{Y}\right) \\
+h\left(\tilde{D} b_{0}\right) \otimes f\left(t_{0}, y_{0}, \mathbf{0}\right)
\end{gathered}
$$

Firstly, we show that the existence of equation (4.16)'s solution. For this, we introduce function $G: \mathbb{R}^{N d} \rightarrow \mathbb{R}^{N d}$ as follows:

$$
G(U)=\mathcal{A B} \mathcal{B}^{T} U+\left(\tilde{D} a_{0}\right) \otimes y_{0}-h \mathcal{B}\left(\hat{D}^{-1} \otimes I_{d}\right) F\left(\left(\hat{D} \otimes I_{d}\right) \mathcal{B}^{T} U, q_{0} \otimes y_{0}+\left((Q \hat{D}) \otimes I_{d}\right) \mathcal{B}^{T} U\right)
$$




$$
-h\left(\tilde{D} b_{0}\right) \otimes f\left(t_{0}, y_{0}, \mathbf{0}\right), \quad U \in \mathbb{R}^{N d} .
$$

In terms of Theorem 6.4.4 in [31], if we assume that equation (4.16) has its solution, it suffices to prove that $G(U)$ is uniformly monotone. Namely, we need only to show that there exists a constant $\eta>0$ such that

$$
\langle G(U)-G(\tilde{U}), U-\tilde{U}\rangle_{h} \geq \eta\|U-\tilde{U}\|_{h}^{2}, \quad \forall U, \tilde{U} \in \mathbb{R}^{N d} .
$$

In fact, it follows from condition $\mathcal{H}$, Lemma 4.1 and the properties of inner product that

$$
\begin{aligned}
& \langle G(U)-G(\tilde{U}), U-\tilde{U}\rangle_{h} \\
= & \left\langle\mathcal{A} \mathcal{B}^{T}(U-\tilde{U}), U-\tilde{U}\right\rangle_{h}-h\left\langle\mathcal{B}\left(\hat{D}^{-1} \otimes I_{d}\right) F\left(\left(\hat{D} \otimes I_{d}\right) \mathcal{B}^{T} U, q_{0} \otimes y_{0}+\left((Q \hat{D}) \otimes I_{d}\right) \mathcal{B}^{T} U\right)\right. \\
& \left.\quad-\mathcal{B}\left(\hat{D}^{-1} \otimes I_{d}\right) F\left(\left(\hat{D} \otimes I_{d}\right) \mathcal{B}^{T} \tilde{U}, q_{0} \otimes y_{0}+\left((Q \hat{D}) \otimes I_{d}\right) \mathcal{B}^{T} \tilde{U}\right), U-\tilde{U}\right\rangle_{h} \\
= & \left\langle\mathcal{A} \mathcal{B}^{T}(U-\tilde{U}), U-\tilde{U}\right\rangle_{h}-h\left\langle\left(\hat{D}^{-2} \otimes I_{d}\right) F\left(\left(\hat{D} \otimes I_{d}\right) \mathcal{B}^{T} U, q_{0} \otimes y_{0}+\left((Q \hat{D}) \otimes I_{d}\right) \mathcal{B}^{T} U\right)\right. \\
& \left.\quad-\left(\hat{D}^{-2} \otimes I_{d}\right) F\left(\left(\hat{D} \otimes I_{d}\right) \mathcal{B}^{T} \tilde{U}, q_{0} \otimes y_{0}+\left((Q \hat{D}) \otimes I_{d}\right) \mathcal{B}^{T} \tilde{U}\right),\left(\hat{D} \otimes I_{d}\right) \mathcal{B}^{T}(U-\tilde{U})\right\rangle_{h} \\
\geq & \left\langle\mathcal{A} \mathcal{B}^{T}(U-\tilde{U}), U-\tilde{U}\right\rangle_{h}-h \mu\left\langle\left(\hat{D}^{-1} \otimes I_{d}\right) \mathcal{B}^{T}(U-\tilde{U}),\left(\hat{D} \otimes I_{d}\right) \mathcal{B}^{T}(U-\tilde{U})\right\rangle_{h} \\
= & \left\langle\mathcal{A} \mathcal{B}^{T}(U-\tilde{U}), U-\tilde{U}\right\rangle_{h}-h \mu\left\langle\mathcal{B} \mathcal{B}^{T}(U-\tilde{U}), U-\tilde{U}\right\rangle_{h} \\
= & \left\langle\left(\frac{\mathcal{A B}^{T}+\mathcal{B} \mathcal{A}^{T}}{2}-h \mu \mathcal{B B} \mathcal{B}^{T}\right)(U-\tilde{U}), U-\tilde{U}\right\rangle_{h} \\
\geq & S(h)\|U-\tilde{U}\|_{h}^{2} .
\end{aligned}
$$

Since $S(h)$ is a positive bounded function on $\left(0, h_{0}\right],(4.17)$ holds and hence the existence of the solution of the extended BVM (3.8) is proven.

The uniqueness of the solution of (3.8) can be shown with a direct application of Theorem 4.1 when setting $\delta=0$. Hence the proof is completed.

\section{Convergence of the Extended BVMs}

This section will deal with convergence of the extended BVMs. An extended BVM (3.8) is called convergent of order $p$ if it has global error $\|\bar{Y}-Y\|_{h}=\mathcal{O}\left(h^{p}\right)$. A convergence theorem of methods (3.8) can be stated as follows.

Theorem 5.1. Assume that conditions (4.1), (4.2) and $\mathcal{H}$ hold and the extended BVM (3.8) has consistent order $q$. Then, when method (3.8) is applied to problem (1.1) with $y(t) \in$ $C^{(m+1)}\left(\left[t_{0}, T\right]\right)$, the derived numerical solution $Y$ is convergent of order $\min \{q, m-\gamma+1\}$.

Proof. Let $\hat{\delta}=h\left(B \otimes I_{d}\right)[F(\bar{Y}, \bar{Z})-F(\bar{Y}, \tilde{Z})], \tilde{Z}=\left(\tilde{z}_{1}^{T}, \ldots, \tilde{z}_{N}^{T}\right)^{T}$ and

$$
\tilde{z}_{n}= \begin{cases}\frac{h^{-\gamma}}{\Gamma(1-\gamma)} \sum_{i=0}^{m} \theta_{i, n}^{m} y\left(t_{i}\right), & 1 \leq n \leq m, \\ \frac{h^{-\gamma}}{\Gamma(1-\gamma)} \sum_{i=0}^{n} \theta_{i, n}^{m} y\left(t_{i}\right), & m<n \leq N,\end{cases}
$$


Taking use of the above symbols and (3.9), we have

$$
\left(A \otimes I_{d}\right) \bar{Y}+a_{0} \otimes y\left(t_{0}\right)=h\left(B \otimes I_{d}\right) F(\bar{Y}, \tilde{Z})+h b_{0} \otimes f\left(t_{0}, y\left(t_{0}\right), \mathbf{0}\right)+\tilde{\delta}+\hat{\delta} .
$$

It follows from Theorem 2.1 that there exists a constant $c_{0}>0$ such that

$$
\left\|z\left(t_{n}\right)-\tilde{z}_{n}\right\|_{\infty} \leq c_{0} h^{m-\gamma+1}, \quad 1 \leq n \leq N .
$$

This, together with condition (4.2) and $N h=T-t_{0}$, implies that

$$
\begin{aligned}
\|\hat{\delta}\|_{h} & \leq h\|B\|_{h}\|F(\bar{Y}, \bar{Z})-F(\bar{Y}, \tilde{Z})\|_{h} \\
& =h\|B\|_{h} \sqrt{h \sum_{i=1}^{N}\left\|f\left(t_{i}, y\left(t_{i}\right), z\left(t_{i}\right)\right)-f\left(t_{i}, y\left(t_{i}\right), \tilde{z}_{i}\right)\right\|_{2}^{2}} \\
& \leq h\|B\|_{h} L_{2} \sqrt{h \sum_{i=1}^{N}\left\|z\left(t_{i}\right)-\tilde{z}_{i}\right\|_{2}^{2}} \leq c_{0} L_{2}\|B\|_{h} \sqrt{N d} h^{m-\gamma+\frac{5}{2}} \\
& =c_{0} L_{2}\|B\|_{h} \sqrt{d\left(T-t_{0}\right)} h^{m-\gamma+2} .
\end{aligned}
$$

Subtracting (3.8) from (5.1) yields

$$
\left(A \otimes I_{d}\right)(\bar{Y}-Y)=h\left(B \otimes I_{d}\right)[F(\bar{Y}, \tilde{Z})-F(Y, Z)]+\tilde{\delta}+\hat{\delta} .
$$

Applying Theorem 4.1 to (5.4) yields for all $h \in\left(0, h_{0}\right]$ that

$$
\|\bar{Y}-Y\|_{h} \leq\left[\frac{\|\hat{D}\|_{h}\left\|\mathcal{B}^{T}\right\|_{h}\|D\|_{h}}{S(h)}\right]\|\tilde{\delta}+\hat{\delta}\|_{h} \leq\left[\frac{\|\hat{D}\|_{h}\left\|\mathcal{B}^{T}\right\|_{h}\|D\|_{h}}{S(h)}\right]\left(\|\tilde{\delta}\|_{h}+\|\hat{\delta}\|_{h}\right) .
$$

Also, since by hypothesis $\mathcal{H}$ that $S(h)$ is a positive bounded function $S(h)$ on $\left(0, h_{0}\right]$, there exist constants $c_{1}>0$ and $h_{1} \in\left(0, h_{0}\right]$ such that

$$
S(h) \geq c_{1} h, \quad \forall h \in\left(0, h_{1}\right]
$$

Moreover, the $q$-order consistency of the method implies that there exists a constant $c_{2}>0$ such that

$$
\|\tilde{\delta}\|_{h} \leq c_{2} h^{q+1}
$$

Therefore, a combination of (5.3), (5.5)-(5.7) concludes that the extended BVM (3.8) is convergent of order $\min \{q, m-\gamma+1\}$. This completes the proof.

Remark 5.1. In Theorem 5.1, in order to assure that the convergence order of numerical solution $Y$ can arrive at $\min \{q, m-\gamma+1\}$, we ask that $y(t) \in C^{(m+1)}\left(\left[t_{0}, T\right]\right)(m \geq 1)$. The same assumption can be seen in $[8,22,27]$ and the references therein. However, this type of strong smooth assumption could not be satisfied at initial point $t_{0}$ for some realistic problems of the form (1.1). Hence, Theorem 5.1 is only applicable to the problems with smooth initial data. As to the high-order convergence condition for the case of nonsmooth initial data, it keeps unknown at present because of the lack of analytical techniques. A similar open issue was also proposed in Ford \& Yan [13]. Although it is difficult to give a high-order convergence criterion for the problems with nonsmooth initial data, luckily, some numerical treatment methods have 
been suggested (see e.g. [23]). Inspired by the idea in Li, Liang \& Yan [23], in the following, we will adopt a technique to improve the computational accuracy of methods (3.8) when the problem has the nonsmooth initial data. Firstly, we divide the subinterval $\left[t_{0}, t_{m}\right](m \geq 1)$ by the equispaced nodes with stepsize $\tilde{h}$ :

$$
t_{0}=t_{m}^{(0)}<t_{m}^{(1)}<\cdots<t_{m}^{\left(n_{1}\right)}=t_{m}, \quad \text { where } n_{1}=\left\lfloor m h^{1-\frac{\min \{q, m-\gamma+1\}}{2-\gamma}}\right\rfloor,
$$

which leads to $\mathcal{O}\left(\tilde{h}^{2-\gamma}\right) \approx \mathcal{O}\left(h^{\min \{q, m-\gamma+1\}}\right)$. Secondly, we apply the second-order extended trapezoidal rule (cf. [4]) and the piecewise linear interpolation to problem (1.1) on $\left[t_{0}, t_{m}\right]$. It is well-known that the piecewise linear interpolation for Caputo derivative (2.3) is just $L 1$ method (cf. [30]) and its convergence order is $\mathcal{O}\left(\tilde{h}^{2-\gamma}\right)$ (cf. [22]). Hence we have that

$$
\left\|\tilde{y}_{m}-y\left(t_{m}\right)\right\|_{h}=\mathcal{O}\left(\tilde{h}^{2-\gamma}\right), \quad \text { where } \tilde{y}_{m} \approx y\left(t_{m}\right) .
$$

Then, we choose $\tilde{y}_{m}$ as the computational initial value and apply the extended BVMs (3.8) to problem (1.1) on $\left[t_{m}, T\right]$. In this way, the numerical solution $Y$ can arrive at the theoretical accuracy $\mathcal{O}\left(h^{\min \{q, m-\gamma+1\}}\right)$.

\section{Numerical Experiments}

In this section, to illustrate the computational efficiency, accuracy and comparability of the extended BVMs, we present several numerical examples. Combining the underlying BVMs: second-order ETR, third-order GBDF and fourth-order $\mathrm{ETR}_{2}$ in Brugnano \& Trigiante [4] with $m$-th $(m=1,2,3)$ interpolation (3.7) for the Caputo derivatives, respectively, we can obtain a series of extended BVMs for problems (1.1). For convenience, correspondingly, we write these extended BVMs as $\operatorname{ETR}(2,1), \operatorname{GBDF}(3,2)$ and $\operatorname{ETR}_{2}(4,3)$. Moreover, in order to show the computational advantages of the extended BVMs, we will consider an adapted version of the product integration methods (PIMs) in $[28,29]$ for $(1.1)$. The adapted $k$-step $(k+1-\gamma)$-order PIMs $(\operatorname{APIM}(k))$ can be expressed as follows:

$$
\sum_{l=1}^{k+1} \frac{\nabla^{l} y_{n}}{l}=h f\left(t_{n}, y_{n}, z_{n}\right), \quad z_{n}=\frac{h^{-\gamma}}{\Gamma(1-\gamma)} \sum_{l=1}^{k} \sum_{j=k+1}^{n} a_{l}(n-j) \nabla^{l} y_{n}, \quad k+1 \leq n \leq N,
$$

where $\nabla$ is the backward difference operator and

$$
a_{l}(n-j)=\int_{-1}^{0} \frac{1}{(n-j-\xi)^{\gamma} l !} \frac{d}{d \xi}[\xi(\xi+1) \cdots(\xi+l-1)] d \xi .
$$

For the implementation of $\operatorname{APIM}(\mathrm{k})$, we will use some $(k+1)$-order one-step methods to get its starting values. Besides the comparison with $\operatorname{APIM}(\mathrm{k})$, we will also consider another class of extended BVMs, which are constructed by combining the underlying BVMs and Li et al's interpolation scheme (7) in [22]. For example, we can combine the second-order ETR, thirdorder GBDF and fourth-order $\mathrm{ETR}_{2}$ with $\mathrm{Li}$ et al's interpolation scheme with $m=1,2,3$ and write the derived methods as $\operatorname{AETR}(2,1), \operatorname{AGBDF}(3,2)$ and $\operatorname{AETR}_{2}(4,3)$, respectively. In the following, global error and convergence order of the above methods will be computed respectively by the formulas:

$$
\operatorname{err}(h):=\|\bar{Y}-Y\|_{h}, \quad \hat{p}:=\log _{2}\left[\frac{\operatorname{err}(h)}{\operatorname{err}(h / 2)}\right] .
$$


Table 6.1: Global errors and convergence orders of $\operatorname{ETR}(2,1), \operatorname{GBDF}(3,2)$ and $\operatorname{ETR}_{2}(4,3)$ for problem $(6.2)$.

\begin{tabular}{|c|c|c|c|c|c|c|}
\hline \multirow[b]{2}{*}{$h$} & \multicolumn{2}{|c|}{$\operatorname{ETR}(2,1)$} & \multicolumn{2}{|c|}{$\operatorname{GBDF}(3,2)$} & \multicolumn{2}{|c|}{$\operatorname{ETR}_{2}(4,3)$} \\
\hline & $\operatorname{err}(h)$ & $\hat{p}$ & $\operatorname{err}(h)$ & $\hat{p}$ & $\operatorname{err}(h)$ & $\hat{p}$ \\
\hline $1 / 2$ & $8.3408 \mathrm{e}-2$ & - & $3.1622 \mathrm{e}-2$ & - & $1.6331 \mathrm{e}-2$ & - \\
\hline $1 / 4$ & $3.2184 \mathrm{e}-2$ & 1.3738 & $6.8565 \mathrm{e}-3$ & 2.2054 & $1.7017 \mathrm{e}-3$ & 3.2625 \\
\hline $1 / 8$ & $1.2028 \mathrm{e}-2$ & 1.4199 & $1.1524 \mathrm{e}-3$ & 2.5728 & $1.4692 \mathrm{e}-4$ & 3.5339 \\
\hline $1 / 16$ & $4.4184 \mathrm{e}-3$ & 1.4448 & $2.2832 \mathrm{e}-4$ & 2.3355 & $1.1208 \mathrm{e}-5$ & 3.7124 \\
\hline
\end{tabular}

Table 6.2: Global errors and convergence orders of $\operatorname{APIM}(k)(k=1,2,3)$ for problem $(6.2)$.

\begin{tabular}{|c|c|c|c|c|c|c|}
\hline \multirow[b]{2}{*}{$h$} & \multicolumn{2}{|c|}{$\operatorname{APIM}(1)$} & \multicolumn{2}{|c|}{$\operatorname{APIM}(2)$} & \multicolumn{2}{|c|}{$\operatorname{APIM}(3)$} \\
\hline & $\operatorname{err}(h)$ & $\hat{p}$ & $\operatorname{err}(h)$ & $\hat{p}$ & $\operatorname{err}(h)$ & $\hat{p}$ \\
\hline $1 / 2$ & $1.7484 \mathrm{e}-1$ & - & $3.2093 \mathrm{e}-2$ & - & $3.1642 \mathrm{e}-2$ & - \\
\hline $1 / 4$ & $6.5514 \mathrm{e}-2$ & 1.4162 & $7.6183 \mathrm{e}^{-}-3$ & 2.0747 & $7.2058 \mathrm{e}-3$ & 2.1346 \\
\hline $1 / 8$ & $2.5109 \mathrm{e}-2$ & 1.3836 & $2.6825 \mathrm{e}^{-}-3$ & 1.5059 & $1.9667 \mathrm{e}-3$ & 1.8734 \\
\hline $1 / 16$ & $9.5292 \mathrm{e}-3$ & 1.3978 & $1.1017 \mathrm{e}^{-}-3$ & 1.2838 & $6.7490 \mathrm{e}-4$ & 1.5430 \\
\hline
\end{tabular}

Table 6.3: Global errors and convergence orders of $\operatorname{AETR}(2,1), \operatorname{AGBDF}(3,2)$ and $\operatorname{AETR}_{2}(4,3)$ for problem (6.2).

\begin{tabular}{|c|c|c|c|c|c|c|}
\hline \multirow[b]{2}{*}{$h$} & \multicolumn{2}{|c|}{$\operatorname{AETR}(2,1)$} & \multicolumn{2}{|c|}{$\operatorname{AGBDF}(3,2)$} & \multicolumn{2}{|c|}{$\operatorname{AETR}_{2}(4,3)$} \\
\hline & $\operatorname{err}(h)$ & $\hat{p}$ & $\operatorname{err}(h)$ & $\hat{p}$ & $\operatorname{err}(h)$ & $\hat{p}$ \\
\hline $1 / 2$ & $8.3408 \mathrm{e}-2$ & - & $3.8400 \mathrm{e}-2$ & - & $1.7947 \mathrm{e}-2$ & - \\
\hline $1 / 4$ & $3.2184 \mathrm{e}-2$ & 1.3738 & $8.0270 \mathrm{e}-3$ & 2.2582 & $1.9063 \mathrm{e}-3$ & 3.2349 \\
\hline $1 / 8$ & $1.2028 \mathrm{e}-2$ & 1.4199 & $1.5110 \mathrm{e}-3$ & 2.4094 & $2.0790 \mathrm{e}-4$ & 3.1968 \\
\hline $1 / 16$ & $4.4184 \mathrm{e}^{-}-3$ & 1.4448 & $3.4198 \mathrm{e}-4$ & 2.1435 & $3.2784 \mathrm{e}^{-5}$ & 2.6648 \\
\hline
\end{tabular}

Example 6.1. Consider the nonlinear problem of CFDEs:

$$
y^{\prime}(t)=-\frac{y^{2}(t)}{1+y^{2}(t)}+\frac{1}{5} \cos \left({ }_{0}^{C} D_{t}^{0.5} y(t)\right)+g(t), \quad t \in[0,3] ; \quad y(0)=0,
$$

where $g(t)$ is a given function such that problem (6.2) has the exact solution $y(t)=t \sqrt{t}$.

Taking stepsizes $h=1 / 2^{i}(i=1,2,3,4)$ and then applying $\operatorname{ETR}(2,1), \operatorname{GBDF}(3,2)$ and $\operatorname{ETR}_{2}(4,3)$ to problem $(6.2)$, respectively, we can obtain a series of numerical solutions of (6.2), where, for attaining the theoretical accuracy presented in Theorem 5.1, we also use the algorithm in Remark 5.1 to cope with the nonsmooth initial condition. As an example, the numerical solution $y_{n}$ solved by $\operatorname{ETR}_{2}(4,3)$ with $h=1 / 16$ is plotted in Fig. 6.1(a), and the error $\varepsilon_{n}=\left|y\left(t_{n}\right)-y_{n}\right|$ is shown in Fig. 6.1(b). A detailer description on the global errors and convergence orders of the above extended BVMs for (6.2) is displayed in Table 6.1. These numerical results confirm the computational effectiveness of the extended BVMs and the theoretical accuracy shown in Theorem 5.1.

In order to exhibit the computational advantage of the extended BVMs, we further apply $\operatorname{APIM}(k)(k=1,2,3), \operatorname{AETR}(2,1), \operatorname{AGBDF}(3,2)$ and $\operatorname{AETR}_{2}(4,3)$ with $h=1 / 2^{i}(i=$ $1,2,3,4)$ to problem $(6.2)$, where the nonsmooth initial condition is treated by an algorithm similar to Remark 5.1. The errors and convergence orders of the above methods are listed in Tables 6.2-6.3. By comparing the numerical results in Tables 6.1-6.3, we can find that, 


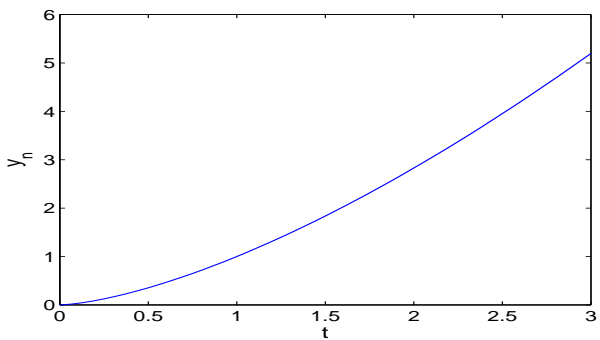

(a)

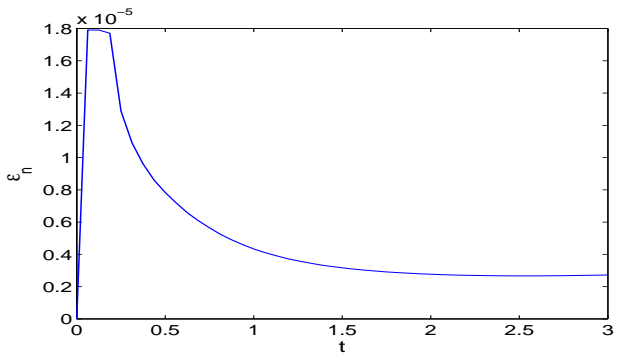

(b)

Fig. 6.1. (a) Numerical solution of problem $(6.2)$ by $\operatorname{ETR}_{2}(4,3)$ with $h=1 / 16$; (b) Error of $\operatorname{ETR}_{2}(4$, 3) with $h=1 / 16$ for problem (6.2).

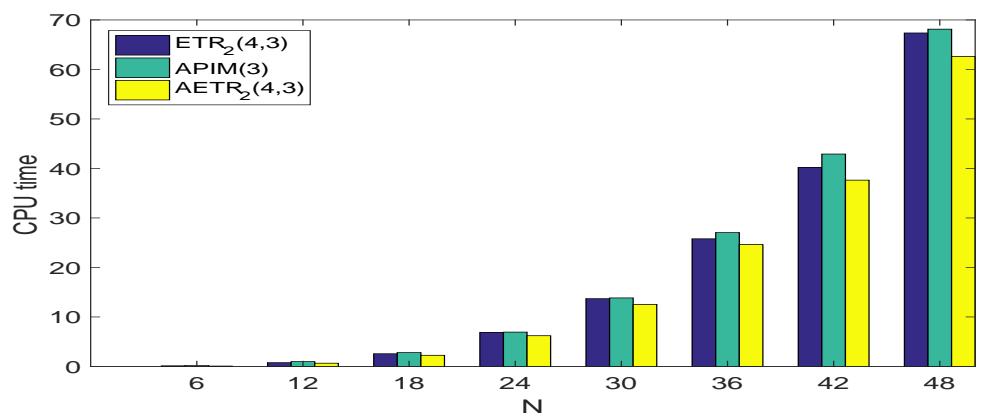

Fig. 6.2. The CPU times (in seconds) of $\operatorname{ETR}_{2}(4,3), \operatorname{APIM}(3)$ and $\operatorname{AETR}_{2}(4,3)$ with $N=(i+1) 6(i=$ $0,1, \ldots, 7)$ for problem $(6.2)$.

when the stepsize is small enough, the extended BVMs (3.8) have higher accuracy than the other two classes methods with the same order, and $\operatorname{APIM}(k)$ have the phenomenon of order reduction. Moreover, we also plot the $\operatorname{CPU}$ times of $\operatorname{ETR}_{2}(4,3), \operatorname{APIM}(3)$ and $\operatorname{AETR}_{2}(4$, $3)$ with $N=(i+1) 6(i=0,1, \ldots, 7)$ for problem (6.2) in Fig. 6.2, which shows that, in computational efficiency, the extended BVMs (3.8) is not better than these with Li et al's interpolation scheme but better than $\operatorname{APIM}(k)$.

Example 6.2. Consider the following fractal mobile/immobile transport models (cf. [26, 33]):

$$
\left\{\begin{array}{l}
\frac{\partial u(x, t)}{\partial t}+{ }_{0}^{C} D_{t}^{\gamma} u(x, t)=\frac{\partial^{2} u(x, t)}{\partial x^{2}}+f(u)+g(x, t), \quad(x, t) \in[0,1] \times[0,3], \\
u(x, 0)=0, \quad x \in[0,1] \\
u(0, t)=u(1, t)=0, \quad t \in[0,3]
\end{array}\right.
$$

where $\gamma \in(0,1), f(u)=u\left(1-u^{2}\right)$ and $g(x, t)$ is a given function such that problem (6.3) has the exact solution $u(x, t)=[x(1-x)]^{4} \sin (\pi t)$. In the following, we will adopt the method of lines to solve this problem.

Firstly, similar to the discretization idea for delay-reaction-diffusion equations in paper [20], we apply a compact difference scheme to discretize the space variable $x$. Let $M$ be a positive integer, $x_{i}=i \Delta x(i=0,1, \ldots, M)$ spatial grid-points with stepsize $\Delta x=1 / M$, and $\mathcal{W}:=\left\{v_{i}: 0 \leq i \leq M\right\}$ grid function space on grid set $\Omega_{\Delta x}:=\left\{x_{i}: 0 \leq i \leq M\right\}$. Define the 


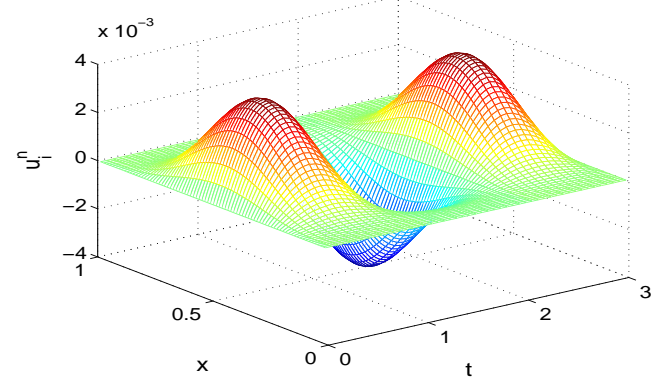

(a)

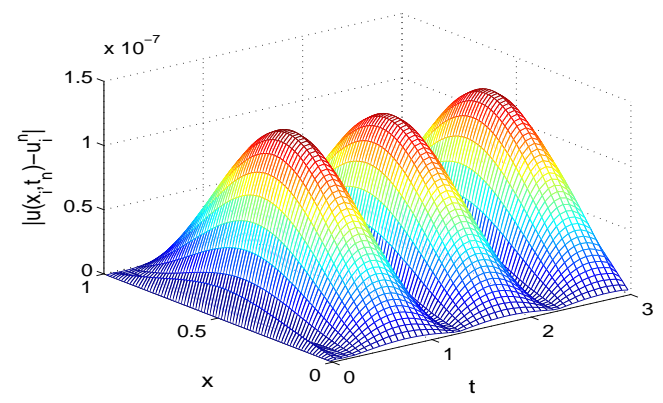

(b)

Fig. 6.3. (a) Numerical solution of problem (6.3) with $\gamma=0.5$ by $\operatorname{ETR}_{2}(4,3)$ with $h=1 / 16$ and $\Delta x=1 / 80$; (b) Error of $\operatorname{ETR}_{2}(4,3)$ with $h=1 / 16$ and $\Delta x=1 / 80$ for problem (6.3) with $\gamma=0.5$.

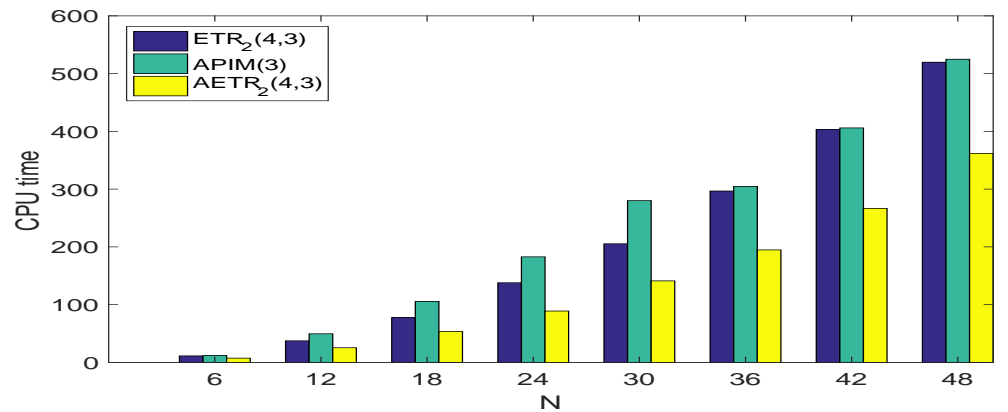

Fig. 6.4. The CPU times (in seconds) of $\operatorname{ETR}_{2}(4,3), \operatorname{APIM}(3)$ and $\operatorname{AETR}_{2}(4,3)$ with $\Delta x=1 / 80$ and $N=(i+1) 6(i=0,1, \ldots, 7)$ for problem $(6.3)$ with $\gamma=0.5$.

following difference operators:

$$
\delta_{x}^{2} v_{i}=\frac{1}{\Delta x^{2}}\left(v_{i+1}-2 v_{i}+v_{i-1}\right), \quad \mathcal{D} v_{i}=\frac{1}{12}\left(v_{i+1}+10 v_{i}+v_{i-1}\right) .
$$

Then a compact difference scheme for (6.3) can be obtained as follows:

$$
\mathcal{D} \frac{\partial u_{i}(t)}{\partial t}+\mathcal{D}_{0}^{C} D_{t}^{\gamma} u_{i}(t)=\delta_{x}^{2} u_{i}(t)+\mathcal{D} f\left(u_{i}(t)\right)+\mathcal{D} g\left(x_{i}, t\right), \quad u_{i}(0)=0, \quad 1 \leq i \leq M,
$$

where $u_{i}(t)$ is an approximation to $u\left(x_{i}, t\right)$. When the following notations are introduced:

$$
\begin{gathered}
Q(t)=\left(\begin{array}{c}
f\left(u_{1}(t)\right)+g\left(x_{1}, t\right) \\
f\left(u_{2}(t)\right)+g\left(x_{2}, t\right) \\
\vdots \\
f\left(u_{M-2}(t)\right)+g\left(x_{M-2}, t\right) \\
f\left(u_{M-1}(t)\right)+g\left(x_{M-1}, t\right)
\end{array}\right), q(t)=\frac{1}{12}\left(\begin{array}{c}
f\left(u_{0}(t)\right)+g\left(x_{0}, t\right) \\
0 \\
\vdots \\
\\
f\left(u_{M}(t)\right)+g\left(x_{M}, t\right)
\end{array}\right), \\
P=\left[\begin{array}{ccccc}
\frac{5}{6} & \frac{1}{12} & 0 & \cdots & 0 \\
\frac{1}{12} & \frac{5}{6} & \frac{1}{12} & \cdots & 0 \\
\vdots & \ddots & \ddots & \ddots & \vdots \\
0 & \cdots & \frac{1}{12} & \frac{5}{6} & \frac{1}{12} \\
0 & \cdots & 0 & \frac{1}{12} & \frac{5}{6}
\end{array}\right], J=\left[\begin{array}{ccccc}
-2 & 1 & 0 & \ldots & 0 \\
1 & -2 & 1 & \ldots & 0 \\
\vdots & \ddots & \ddots & \ddots & \vdots \\
0 & \cdots & 1 & -2 & 1 \\
0 & \cdots & 0 & 1 & -2
\end{array}\right] \in \mathbb{R}^{(M-1) \times(M-1)},
\end{gathered}
$$


Table 6.4: Global errors and convergence orders of $\operatorname{ETR}(2,1), \operatorname{GBDF}(3,2)$ and $\operatorname{ETR}_{2}(4,3)$ with $\Delta x=1 / 80$ for problems $(6.3)$ with $\gamma=0.25,0.5,0.75$, respectively.

\begin{tabular}{|c|c|c|c|c|c|c|c|}
\hline \multirow[b]{2}{*}{$\gamma$} & \multirow[b]{2}{*}{$h$} & \multicolumn{2}{|c|}{$\operatorname{ETR}(2,1)$} & \multicolumn{2}{|c|}{$\operatorname{GBDF}(3,2)$} & \multicolumn{2}{|c|}{$\operatorname{ETR}_{2}(4,3)$} \\
\hline & & $\operatorname{err}(h)$ & $\hat{p}$ & $\operatorname{err}(h)$ & $\hat{p}$ & $\operatorname{err}(h)$ & $\hat{p}$ \\
\hline \multirow[t]{4}{*}{0.25} & $1 / 2$ & $1.6977 \mathrm{e}-3$ & - & $2.3258 \mathrm{e}-3$ & - & $6.4404 \mathrm{e}^{-4}$ & - \\
\hline & $1 / 4$ & $3.2183 \mathrm{e}-4$ & 2.3992 & $2.9792 \mathrm{e}-4$ & 2.9647 & $4.6988 \mathrm{e}^{-5}$ & 3.7768 \\
\hline & $1 / 8$ & $8.1896 \mathrm{e}-5$ & 1.9744 & $3.4452 \mathrm{e}-5$ & 3.1123 & $3.2047 \mathrm{e}-6$ & 3.8740 \\
\hline & $1 / 16$ & $2.2643 \mathrm{e}^{-5}$ & 1.8547 & $4.3265 \mathrm{e}-6$ & 2.9933 & $2.0667 \mathrm{e}^{-}-7$ & 3.9548 \\
\hline \multirow[t]{4}{*}{0.5} & $1 / 2$ & $1.5969 \mathrm{e}-3$ & - & $2.4827 \mathrm{e}-3$ & - & $1.1515 \mathrm{e}-3$ & - \\
\hline & $1 / 4$ & $4.7657 \mathrm{e}-4$ & 1.7445 & $3.7071 \mathrm{e}-4$ & 2.7436 & $1.1528 \mathrm{e}-4$ & 3.3202 \\
\hline & $1 / 8$ & $1.7872 \mathrm{e}-4$ & 1.4150 & $4.9302 \mathrm{e}-5$ & 2.9106 & $1.0843 \mathrm{e}^{-5}$ & 3.4103 \\
\hline & $1 / 16$ & $6.6121 \mathrm{e}^{-5}$ & 1.4345 & $7.3897 \mathrm{e}-6$ & 2.7381 & $9.5079 \mathrm{e}^{-7}$ & 3.5116 \\
\hline \multirow[t]{4}{*}{0.75} & $1 / 2$ & $2.2629 \mathrm{e}-3$ & - & $2.9855 \mathrm{e}-3$ & - & $2.0329 \mathrm{e}-3$ & - \\
\hline & $1 / 4$ & $1.0753 \mathrm{e}-3$ & 1.0734 & $6.0230 \mathrm{e}-4$ & 2.3094 & $2.8450 \mathrm{e}-4$ & 2.8371 \\
\hline & $1 / 8$ & $4.8749 \mathrm{e}-4$ & 1.1414 & $1.1175 \mathrm{e}-4$ & 2.4302 & $3.3280 \mathrm{e}^{-5}$ & 3.0957 \\
\hline & $1 / 16$ & $2.1178 \mathrm{e}-4$ & 1.2028 & $2.2659 \mathrm{e}-5$ & 2.3021 & $3.5662 \mathrm{e}-6$ & 3.2222 \\
\hline
\end{tabular}

Table 6.5: Global errors and convergence orders of $\operatorname{APIM}(k)(k=1,2,3)$ with $\Delta x=1 / 80$ for problems (6.3) with $\gamma=0.25,0.5,0.75$, respectively.

\begin{tabular}{|c|c|c|c|c|c|c|c|}
\hline \multirow[b]{2}{*}{$\gamma$} & \multirow[b]{2}{*}{$h$} & \multicolumn{2}{|c|}{$\operatorname{APIM}(1)$} & \multicolumn{2}{|c|}{$\operatorname{APIM}(2)$} & \multicolumn{2}{|c|}{$\operatorname{APIM}(3)$} \\
\hline & & $\operatorname{err}(h)$ & $\hat{p}$ & $\operatorname{err}(h)$ & $\hat{p}$ & $\operatorname{err}(h)$ & $\hat{p}$ \\
\hline \multirow[t]{4}{*}{0.25} & $1 / 2$ & $3.3106 \mathrm{e}-3$ & - & $3.0310 \mathrm{e}-3$ & - & $3.5194 \mathrm{e}^{-}-3$ & - \\
\hline & $1 / 4$ & $1.0885 \mathrm{e}-3$ & 1.6048 & $6.9830 \mathrm{e}-4$ & 2.1179 & $3.8015 \mathrm{e}-4$ & 3.2107 \\
\hline & $1 / 8$ & $2.9990 \mathrm{e}-4$ & 1.8597 & $9.6552 \mathrm{e}-5$ & 2.8545 & $2.5729 \mathrm{e}-5$ & 3.8851 \\
\hline & $1 / 16$ & $7.7447 \mathrm{e}-5$ & 1.9532 & $1.2263 \mathrm{e}-5$ & 2.9770 & $1.7029 \mathrm{e}-6$ & 3.9173 \\
\hline \multirow[t]{4}{*}{0.5} & $1 / 2$ & $3.5919 \mathrm{e}-3$ & - & $3.1167 \mathrm{e}-3$ & - & $3.6611 \mathrm{e}^{-3}$ & - \\
\hline & $1 / 4$ & $1.1938 \mathrm{e}-3$ & 1.5891 & $7.3156 \mathrm{e}-4$ & 2.0910 & $4.0402 \mathrm{e}^{-4}$ & 3.1798 \\
\hline & $1 / 8$ & $3.4644 \mathrm{e}-4$ & 1.7849 & $1.0313 \mathrm{e}-4$ & 2.8265 & $2.7769 \mathrm{e}-5$ & 3.8629 \\
\hline & $1 / 16$ & $9.9953 \mathrm{e}-5$ & 1.7933 & $1.3625 \mathrm{e}-5$ & 2.9202 & $1.9315 \mathrm{e}-6$ & 3.8457 \\
\hline \multirow[t]{4}{*}{0.75} & $1 / 2$ & $4.3676 \mathrm{e}-3$ & - & $3.4820 \mathrm{e}-3$ & - & $4.0693 \mathrm{e}^{-}-3$ & - \\
\hline & $1 / 4$ & $1.6370 \mathrm{e}-3$ & 1.4158 & $8.9347 \mathrm{e}-4$ & 1.9624 & $5.0667 \mathrm{e}-4$ & 3.0057 \\
\hline & $1 / 8$ & $5.9337 \mathrm{e}-4$ & 1.4640 & $1.4770 \mathrm{e}-4$ & 2.5968 & $4.2661 \mathrm{e}^{-5}$ & 3.5700 \\
\hline & $1 / 16$ & $2.2884 \mathrm{e}-4$ & 1.3746 & $2.5723 \mathrm{e}-5$ & 2.5215 & $3.9640 \mathrm{e}-6$ & 3.4279 \\
\hline
\end{tabular}

and $y(t)=\left(u_{1}(t), \ldots, u_{M-1}(t)\right)^{T}$, then (6.4) can be written in an equivalent form:

$$
\left\{\begin{array}{l}
y^{\prime}(t)+{ }_{0}^{C} D_{t}^{\gamma} y(t)=\frac{1}{\Delta x^{2}} P^{-1} J y(t)+Q(t)+P^{-1} q(t), \quad t \in[0,3], \\
y(0)=\mathbf{0} \in \mathbb{R}^{M-1}
\end{array}\right.
$$

Secondly, we take stepsizes $\Delta x=1 / 80, h=1 / 2^{i}(i=1,2,3,4)$ and then apply $\operatorname{ETR}(2,1)$, $\operatorname{GBDF}(3,2)$ and $\operatorname{ETR}_{2}(4,3)$ to solve $(6.5)$ with $\gamma=0.25,0.5,0.75$, respectively. In this way, a series of effective numerical solutions for (6.3) can be obtained. For simplicity, in Fig. 6.3(a), we only plot the numerical solution of $(6.3)$ with $\gamma=0.5$ solved by $\operatorname{ETR}_{2}(4,3)$ with $\Delta x=1 / 80$ and $h=1 / 16$, and the global errors $\left|u\left(x_{i}, t_{n}\right)-u_{i}^{n}\right|(0 \leq i \leq 80,0 \leq n \leq 16)$ are plotted in Fig. 6.3(b). A whole description to the global errors and convergence orders of the above 
Table 6.6: Global errors and convergence orders of $\operatorname{AETR}(2,1), \operatorname{AGBDF}(3,2)$ and $\operatorname{AETR}_{2}(4,3)$ with $\Delta x=1 / 80$ for problems $(6.3)$ with $\gamma=0.25,0.5,0.75$, respectively.

\begin{tabular}{|c|c|c|c|c|c|c|c|}
\hline \multirow[b]{2}{*}{$\gamma$} & \multirow[b]{2}{*}{$h$} & \multicolumn{2}{|c|}{$\operatorname{AETR}(2,1)$} & \multicolumn{2}{|c|}{$\operatorname{AGBDF}(3,2)$} & \multicolumn{2}{|c|}{$\operatorname{AETR}_{2}(4,3)$} \\
\hline & & $\operatorname{err}(h)$ & $\hat{p}$ & $\operatorname{err}(h)$ & $\hat{p}$ & $\operatorname{err}(h)$ & $\hat{p}$ \\
\hline \multirow[t]{4}{*}{0.25} & $1 / 2$ & $1.6977 \mathrm{e}-3$ & - & $2.2265 \mathrm{e}-3$ & - & $6.7201 \mathrm{e}^{-4}$ & - \\
\hline & $1 / 4$ & $3.2183 \mathrm{e}-4$ & 2.3992 & $2.9227 \mathrm{e}-4$ & 2.9294 & $5.2910 \mathrm{e}-5$ & 3.6669 \\
\hline & $1 / 8$ & $8.1896 \mathrm{e}-5$ & 1.9744 & $3.6030 \mathrm{e}-5$ & 3.0200 & $4.6995 \mathrm{e}-6$ & 3.4930 \\
\hline & $1 / 16$ & $2.2643 \mathrm{e}-5$ & 1.8547 & $4.6904 \mathrm{e}-6$ & 2.9414 & $3.9299 \mathrm{e}-7$ & 3.5799 \\
\hline \multirow[t]{4}{*}{0.5} & $1 / 2$ & $1.5969 \mathrm{e}-3$ & - & $2.2763 \mathrm{e}-3$ & - & $1.2429 \mathrm{e}-3$ & - \\
\hline & $1 / 4$ & $4.7657 \mathrm{e}-4$ & 1.7445 & $3.5974 \mathrm{e}-4$ & 2.6617 & $1.4566 \mathrm{e}-4$ & 3.0930 \\
\hline & $1 / 8$ & $1.7872 \mathrm{e}-4$ & 1.4150 & $5.4326 \mathrm{e}-5$ & 2.7272 & $1.6634 \mathrm{e}^{-}-5$ & 3.1304 \\
\hline & $1 / 16$ & $6.6121 \mathrm{e}-5$ & 1.4345 & $8.6396 \mathrm{e}-6$ & 2.6526 & $1.6936 \mathrm{e}-6$ & 3.2960 \\
\hline \multirow[t]{4}{*}{0.75} & $1 / 2$ & $2.2629 \mathrm{e}-3$ & - & $2.7745 \mathrm{e}-3$ & - & $2.2374 \mathrm{e}-3$ & - \\
\hline & $1 / 4$ & $1.0753 \mathrm{e}-3$ & 1.0734 & $6.0675 \mathrm{e}-4$ & 2.1930 & $3.6124 \mathrm{e}-4$ & 2.6308 \\
\hline & $1 / 8$ & $4.8749 \mathrm{e}-4$ & 1.1414 & $1.2270 \mathrm{e}-4$ & 2.3060 & $4.8412 \mathrm{e}^{-}-5$ & 2.8995 \\
\hline & $1 / 16$ & $2.1178 \mathrm{e}-4$ & 1.2028 & $2.5055 \mathrm{e}-5$ & 2.2920 & $5.7590 \mathrm{e}-6$ & 3.0715 \\
\hline
\end{tabular}

methods can be seen in Table 6.4. Again, the numerical results verify the computational effectiveness of the extended BVMs (3.8) and their theoretical accuracy shown in Theorem 5.1.

For giving a comparison, in Tables 6.5-6.6, we also present the global errors and convergence orders of $\operatorname{APIM}(k)(k=1,2,3), \operatorname{AETR}(2,1), \operatorname{AGBDF}(3,2)$ and $\operatorname{AETR}_{2}(4,3)$ with stepsizes $\Delta x=1 / 80$ and $h=1 / 2^{i}(i=1,2,3,4)$ for problems $(6.3)$ with $\gamma=0.25,0.5,0.75$, respectively. From Tables 6.4-6.6, we can find that the extend BVMs (3.8) have the best accuracy under the same order and stepsize. Moreover, in Fig. 6.4, we also plot CPU times (in seconds) of $\operatorname{ETR}_{2}(4$, 3), $\operatorname{APIM}(3)$ and $\operatorname{AETR}_{2}(4,3)$ with $\Delta x=1 / 80$ and $N=(i+1) 6(i=0,1, \ldots, 7)$ for problem (6.3) with $\gamma=0.5$. This, again, shows that the computational efficiency of the extended BVMs (3.8) is not better than that of the methods with Li et al's interpolation scheme but better than that of $\operatorname{APIM}(k)$.

Acknowledgments. The authors would like to thank the anonymous referees for their valuable comments and helpful suggestions. The second author Chengjian Zhang (corresponding author) is supported by NSFC (Grant No. 11971010).

\section{References}

[1] L. Aceto, C. Magherini and P. Novati, Generalized Adams methods for fractional differential equations, AIP Conf. Proc., 1479 (2012), 250-253.

[2] L. Aceto, C. Magherini and P. Novati, Fractional convolution quadrature based on generalized Adams methods, Calcolo, 51 (2014), 441-463.

[3] Y.I. Babenko, Heat and Mass Transfer, Khimiya, Leningrad, 1986 (In Russian).

[4] L. Brugnano and D. Trigiante, Solving Differential Problems by Multistep Initial and Boundary Value Methods, Gordan \& Breach, Amsterdam, 1998.

[5] L. Brugnano and D. Trigiante, Boundary value methods: the third way between linear multistep and Runge-Kutta methods, Comput. Math. Appl., 36 (1998), 269-284.

[6] L. Brugnano, C. Zhang and D. Li, A class of energy-conserving Hamiltonian boundary value methods for nonlinear Schrödinger equation with wave operator, Commun. Nonlinear Sci. Numer. Simul., 60 (2018), 33-49. 
[7] H. Brunner and T. Tang, Polynomial spline collocation methods for the nonlinear Basset equation, Comput. Math. Appl., 18 (1989), 449-457.

[8] J. Cao and C. Xu, A high order schema for the numerical solution of the fractional ordinary differential equations, J. Comput. Phys., 238 (2013) 154-168.

[9] W. Cao, F. Zeng, Z. Zhang and G.E. Karniadakis, Implicit-explicit difference schemes for nonlinear fractional differential equations with nonsmooth solutions, SIAM J. Sci. Comput., 38 (2016), 3070-3093.

[10] H. Chen and C. Zhang, Boundary value methods for Volterra integral and integro-differential equations, Appl. Math. Comput., 218 (2011), 2619-2630.

[11] H. Chen and C. Zhang, Block boundary value methods for solving Volterra integral and integrodifferential equations, J. Comput. Appl. Math., 236 (2012), 2822-2837.

[12] H. Chen and C. Zhang, Convergence and stability of extended block boundary value methods for Volterra delay integro-differential equations, Appl. Numer. Math., 62 (2012), 141-154.

[13] N.J. Ford and Y. Yan, An approach to construct higher order time discretisation schemes for time fractional partial differential equations with nonsmooth data, Fract. Calc. Appl. Anal., 20 (2017), 1076-1105.

[14] E. Hairer and P. Maass, Numerical methods for singular nonlinear integro-differential equations, Appl. Numer. Math., 3 (1987), 243-256.

[15] F. Iavernaro and F. Mazzia, Convergence and stability of multistep methods solving nonlinear initial value problems, SIAM J. Sci. Comput., 18 (1997), 270-285.

[16] F. Iavernaro and F. Mazzia, Block-boundary value methods for the solution of ordinary differential equations, SIAM J. Sci. Comput., 21 (1999), 323-339.

[17] B. Jin, B. Li and Z. Zhou, Correction of high-order BDF convolution quadrature for fractional evolution equations, SIAM J. Sci. Comput., 39 (2017), 3129-3152.

[18] C. Li and C. Zhang, Block boundary value methods applied to functional differential equations with piecewise continuous arguments, Appl. Numer. Math., 115 (2017), 214-224.

[19] C. Li and C. Zhang, The extended generalized Störmer-Cowell methods for second-order delay boundary value problems, Appl. Math. Comput., 294 (2017), 87-95.

[20] D. Li, C. Zhang and J. Wen, A note on compact finite difference method for reaction-diffusion equations with delay, Appl. Math. Model., 39 (2015), 1749-1754.

[21] D. Li, J. Zhang and Z. Zhang, Unconditionally optimal error estimates of a linearized Galerkin method for nonlinear time fractional reaction-subdiffusion equations, J. Sci. Comput., 76 (2018), 848-866.

[22] H. Li, J. Cao and C. Li, High-order approximation to Caputo derivatives and Caputo-type advection-diffusion equations (III), J. Comput. Appl. Math., 299 (2016), 159-175.

[23] Z. Li, Z. Liang and Y. Yan, High-order numerical methods for solving time fractional partial differential equations, J. Sci. Comput., 71 (2017), 785-803.

[24] H. Liao, D. Li and J. Zhang, Sharp error estimate of the nonuniform $L 1$ formula for linear reactionsubdiffusion equations, SIAM J. Numer. Anal., 56 (2018), 1112-1133.

[25] Y. Lin, X. Li and C. Xu, Finite difference/spectral approximations for the fractional cable equation, Math. Comput., 80 (2011), 1369-1396.

[26] F. Liu, M.M. Meerschaert, R.J. McGough, P. Zhuang and Q. Liu, Numerical methods for solving the multi-term time-fractional wave-diffusion equation, Fract. Calc. Appl. Anal., 16 (2013), 9-25.

[27] W. Luo, C. Li, T. Huang, X. Gu and G. Wu, A high-order accurate numerical scheme for the Caputo derivative with applications to fractional diffusion problems, Numer. Func. Anal. Opt., 39 (2018), 600-622.

[28] S. McKee, The analysis of a variable step, variable coefficient linear multistep method for solving a singular integro-differential equation arising from the diffusion of discrete particles in a turbulent fluid, IMA J. Appl. Math., 23 (1979), 373-388.

[29] S. McKee and A. Stokes, Product integration methods for the nonlinear Basset equation, SIAM 
J. Numer. Anal., 20 (1983), 143-160.

[30] K. Oldham and J. Spanier, The Fractional Calculus: Theory and Applications of Differentiation and Integration to Arbitrary Order, Academic Press, New York, 1974.

[31] J.M. Ortega and W.C. Rheinboldt, Iterative Solution of Nonlinear Equations in Several Variables, Academic Press, New York, 1970.

[32] I. Podlubny, Fractional Differential Equations, Academic Press, San Diego, 1999.

[33] R. Schumer, D.A. Benson, M.M. Meerschaert and B. Baeumer, Fractal mobile/immobile solute transport, Water Resour. Res., 39 (2003), 1296-1307.

[34] M. Stynes, E. O'Riordan and J.L. Gracia, Error analysis of a finite difference method on graded meshes for a time-fractional diffusion equation, SIAM J. Numer. Anal., 55 (2017), 1057-1079.

[35] E. Süli and D.F. Mayers, An Introduction to Numerical Analysis, Cambridge University Press, Cambridge, 2003.

[36] Z. Sun and G. Gao, Finite Difference Methods for Fractional Differential Equations, Science Press, Beijing, 2016.

[37] H. Wang, C. Zhang and Y. Zhou, A class of compact boundary value methods applied to semilinear reaction-diffusion equations, Appl. Math. Comput., 325 (2018), 69-81.

[38] Y. Xu, J. Zhao and Z. Gao, Stability analysis of block boundary value methods for neutral pantograph equation, J. Diff. Equ. Appl., 19 (2013), 1227-1242.

[39] Y. Xu, J. Zhao and Z. Gao, Stability analysis of block boundary value methods for the neutral differential equation with many delays, Appl. Math. Model., 38 (2014), 325-335.

[40] X. Yan and C. Zhang, Solving nonlinear functional-differential and functional equations with constant delay via block boundary value methods, Math. Comput. Simul., (2019), in Press.

[41] C. Zhang and H. Chen, Block boundary value methods for delay differential equations, Appl. Numer. Math., 60 (2010), 915-923.

[42] C. Zhang, H. Chen and L. Wang, Strang-type preconditioners applied to ordinary and neutral differential-algebraic equations, Numer. Linear Alge. Appl., 18 (2011), 843-855.

[43] C. Zhang and C. Li, Generalized Störmer-Cowell methods for nonlinear BVPs of second-order delay-integro-differential equations, J. Sci. Comput., 74 (2018), 1221-1240.

[44] C. Zhang and H. Wang, Solving nonlinear second-order delay initial value problems via the adapted Generalized Störmer-Cowell methods, Appl. Math. Lett., 96 (2019), 172-178. 